\title{
Free Vibration Analysis of a Free-Free Single-Tapered Beam Carrying Arbitrary Concentrated Elements Using Modified Mode-Superposition Method (MMSM)
}

Chia-Chin Wu

Department of Mechanical and Energy Engineering, National Chia-Yi University, Chia-Yi, Taiwan, joechiachin.wu@mail.ncyu.edu.tw

Follow this and additional works at: https://jmstt.ntou.edu.tw/journal

Part of the Fresh Water Studies Commons, Marine Biology Commons, Ocean Engineering Commons, Oceanography Commons, and the Other Oceanography and Atmospheric Sciences and Meteorology Commons

\section{Recommended Citation}

Wu, Chia-Chin (2021) "Free Vibration Analysis of a Free-Free Single-Tapered Beam Carrying Arbitrary Concentrated Elements Using Modified Mode-Superposition Method (MMSM)," Journal of Marine Science and Technology. Vol. 29: Iss. 3, Article 11.

DOI: $10.51400 / 2709-6998.1441$

Available at: https://jmstt.ntou.edu.tw/journal/vol29/iss3/11

This Research Article is brought to you for free and open access by Journal of Marine Science and Technology. It has been accepted for inclusion in Journal of Marine Science and Technology by an authorized editor of Journal of Marine Science and Technology. 


\title{
Free Vibration Analysis of a Free-Free Single-Tapered Beam Carrying Arbitrary Concentrated Elements Using Modified Mode-Superposition Method (MMSM)
}

\author{
Chia-Chin Wu \\ Department of Mechanical and Energy Engineering, National Chia-Yi University, Chia-Yi, Taiwan
}

\begin{abstract}
The literature contains scant information on the free vibration analyses of the loaded single-tapered beams (STBs) (i.e., STBs carrying concentrated elements (CEs)) Furthermore, even when such studies have been performed, they generally only consider the case of constrained (e.g., free-clamped (F-C)) beams. In other words, the problem of unconstrained (e.g., free-free (F-F)) loaded STBs has not yet to be addressed. Thus, the present study employs the modified modesuperposition method (MMSM) to investigate the free vibration characteristics of the F-F loaded STB carrying arbitrary various CEs. The investigation focuses particularly on the natural frequencies and mode shapes of the rigid-body motions and elastic vibrations of the STB. The presented MMSM differs from the conventional mode-superposition method (CMSM) in that the mode shapes of the rigid-body motions of the F-F bare STB are also taken into account, whereas the CMSM considers only the elastic vibration of the STB. The numerical results show that, for the considered F-F loaded STB problem, the CMSM provides neither any information regarding the rigid-body motions of the beam, nor any satisfactory solutions for the elastic vibrations. The correctness of the presented theory and developed computer code is demonstrated by comparing the numerical results obtained using the proposed MMSM with those obtained from the finite element method (FEM). It is shown that the two sets of results are in good agreements.
\end{abstract}

Keywords: Single-tapered beam (STB), Bare beam, Loaded beam, Rigid-body motions, Elastic vibrations, Modified mode-superposition method (MMSM)

\section{Introduction}

$I_{\mathrm{s}}^{\mathrm{n}}$ n engineering, the vibration characteristics of some structural systems can be evaluated by using those of a uniform or non-uniform beam carrying various concentrated elements (CEs), such as lumped masses, translational springs, rotational springs, and/or spring-mass systems. However, among the many related studies which have been presented, most consider only uniform beams, e.g., $[1,14,17,18,26,33]$; and [25]. In other words, the literature contains relatively few studies on non-uniform beams. Some of these studies are briefly reviewed in introduction of the article presented by [35]. Of those studies which have been performed on non-uniform beams, most focus on the problem of bare beams, i.e., beams carrying no CEs $[2,7,8,13,15,20,21]$; and

Received 5 September 2019; revised 9 December 2019; accepted 22 September 2020.

Available online 25 June 2021.

E-mail address: joechia-chin.wu@mail.ncyu.edu.tw. 
[16]. That is, loaded beams carrying only one or two concentrated elements (CEs) have seldom been discussed [19,22]; and [12]. For uniform or non-uniform loaded beams carrying more than two CEs located at arbitrary positions along the beam length (such as [3,4,9,33,34]; and [35], the vibration problem is complex and intractable. Thus, such problems are most conveniently solved using the mode-superposition method (MSM) [33-35].

Generally speaking, non-uniform beams may be single-tapered or double-tapered, and may have various constrained boundary conditions (BCs), including pinned-pinned (P-P), clamped-clamped (C-C) or free-clamped (F-C). To the best of the author's knowledge, the free vibration of unconstrained free-free (F-F) single-tapered beams (STBs) has not yet been investigated in the literature. The present study addresses this gap using the modified mode-superposition method (MMSM) previously presented by [31,32]. For the problem considered in the present study, the MMSM formulation comprises three parts: (i) determining the exact solutions for the natural frequencies and normal mode shapes of the F-F bare STB associated with elastic vibrations; (ii) determining the exact solutions for the natural frequencies and normal mode shapes of the F-F bare STB associated with rigid-body motions; (iii) obtaining the natural frequencies and mode shapes associated with the coupled rigidbody motions and elastic vibrations of the F-F loaded STB using MSM with the effects of various CEs and rigid-body motions of the F-F bare STB taken into account. Notably, conventional MSM (CMSM) does not consider the effects of rigid-body motions of the F-F bare STB, and hence cannot provide any information regarding the rigid-body motions of the F-F loaded STB or its elastic vibrations. By contrast, all the last drawbacks of the CMSM have been removed from the MMSM. In order to confirm the correctness of the MMSM results, all numerical examples are also solved with the FEM, and it is found that all numerical results obtained from MMSM are in good agreements with those obtained from FEM.

\section{Formulation of MMSM}

This section derives the detailed formalisms of the three parts described above, namely: (i) free elastic vibrations of the F-F bare STB; (ii) free rigid-body motions of the F-F bare STB; and (iii) free elastic vibrations coupling with rigid-body motions of the F-F loaded STB carrying various CEs.

\subsection{Free elastic vibrations of F-F bare STB}

This subsection derives the exact solutions for the natural frequencies and associated normal mode shapes of the F-F bare STB during free elastic vibration. Fig. 1(a)-(c) present the top, front, and leftside views, respectively, of the considered truncated F-F STB. As shown, the breadth of the beam is constant, i.e., $b_{0}=b_{1}=b$, while its height at the small end (located at $x=L_{0}$ ) is $h_{0}$ and that at the large end (located at $x=L_{1}$ ) is $h_{1}$. Finally, the beam length is given by $L=L_{1}-L_{0}$.

For the non-uniform Euler-Bernoulli bare beam shown in Fig. 1, the equation of motion is given by $[7,8,35]$ as

$\frac{\partial^{2}}{\partial x^{2}}\left[E \bar{I}(x) \frac{\partial^{2} u_{y}(x, t)}{\partial x^{2}}\right]+\rho A(x) \frac{\partial^{2} u_{y}(x, t)}{\partial t^{2}}=0$

where $u_{y}(x, t)$ is the transverse deflection of the beam at axial coordinate $x$ and time $t, E$ is the Young's modulus of the beam material, $\rho$ is the mass density. In addition, $A(x)$ is the cross-sectional area of the beam and $\bar{I}(x)$ is the moment of inertia of area $A(x)$ about the $z$-axis.

For free vibrations, one has

$u_{y}(x, t)=W(x) e^{j \omega t}$

where $W(x)$ is the amplitude of the deflection function $u_{y}(x, t), \omega$ is the natural frequency of the bare beam, and $j=\sqrt{-1}$.

From Eqs. (1) and (2), one obtains

$\frac{d^{2}}{d x^{2}}\left[E \bar{I}(x) \frac{d^{2} W(x)}{d x^{2}}\right]-\rho A(x) \omega^{2} W(x)=0$

If the values of $A(x)$ and $\bar{I}(x)$ appearing in Eq. (3) take the forms

$A(x)=A_{1}\left(x / L_{1}\right)^{p}$ and $\bar{I}(x)=\bar{I}_{1}\left(x / L_{1}\right)^{p+2}$

or

$A(\xi)=A_{1} \xi^{p}$ and $\bar{I}(\xi)=\bar{I}_{1} \xi^{p+2}$

with

$\xi=x / L_{1}$

then the solution of Eq. (3) is given by [35]; and [28] as

$W(x)=x^{-p / 2}\left[c_{1} J_{p}(z)+c_{2} Y_{p}(z)+c_{3} I_{p}(z)+c_{4} K_{p}(z)\right]$

In Eqs. (4) and (6), $L_{1}$ is the length of the complete tapered beam from the sharp end (i.e., the origin "o" of the axial coordinate $x$ ) to the large end, 


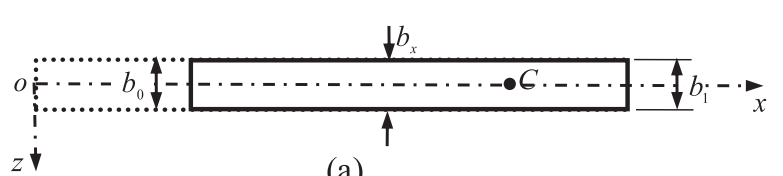

(a)

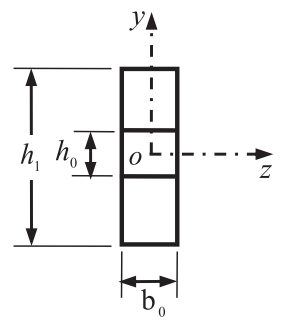

(c)

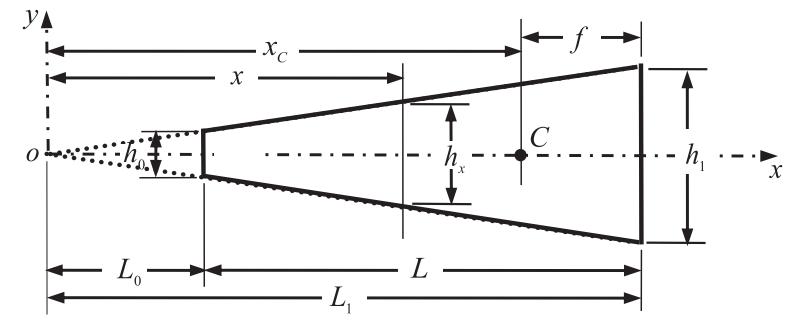

(b)

Fig. 1. Schematic illustrations of truncated F-F single-tapered beam: (a) top view; (b) front view; (c) left-side view. The distance from the center of gravity (c.g.) of the bare beam, $C$, to the large end is given by $f=\left(L_{1} / 3\right)\left(1+\xi_{0}-2 \xi_{0}^{2}\right) /\left(1+\xi_{0}\right)$, where $\xi_{0}=L_{0} / L_{1}$.

while $A_{1}$ and $\bar{I}_{1}$ denote the cross-sectional area and moment of inertia of the large end, respectively. Furthermore, in Eq. (7), $J_{p}$ and $Y_{p}$ are the $p$-th order Bessel functions of the first kind and second kind, respectively, $I_{p}$ and $K_{p}$ are the $p$-th order modified Bessel functions of the first kind and second kind, respectively, and $c_{1} \sim c_{4}$ are integration constants determined by the BCs. In addition,

$z=2 \beta L_{1} \xi^{1 / 2}=2 \beta L_{1}^{1 / 2} x^{1 / 2}$

$\beta^{4}=\omega^{2} \rho A_{1} /\left(E \bar{I}_{1}\right)$

From Fig. 1, the height of the sectional area located at axial position $x$ is given by

$h_{x}=h_{1}\left(x / L_{1}\right)=h_{1} \xi$

Thus, the sectional area, $A(x)$, and sectional moment of inertia, $\bar{I}(x)$, of the STB are given respectively as

$A(x)=b h_{x}=b h_{1} \xi=A_{1} \xi$

$\bar{I}(x)=\frac{1}{12} b h_{x}^{3}=\frac{1}{12} b h_{1}^{3} \xi^{3}=\bar{I}_{1} \xi^{3}$

where

$A_{1}=b h_{1}, \bar{I}_{1}=\frac{1}{12} b h_{1}^{3}$

Comparing Eqs. (11) and (12) with Eqs. (4a,b) or $(5 a, b)$ one finds that, for a STB, the order of the Bessel functions is

$p=1$

Substituting this value of $p$ into Eq. (7) yields
$W(x)=x^{-1 / 2}\left[c_{1} J_{1}(z)+c_{2} Y_{1}(z)+c_{3} I_{1}(z)+c_{4} K_{1}(z)\right]$

where the constants, $c_{1}-c_{4}$, are determined by the $\mathrm{BCs}$ of the beam [28], e.g.,

(i) $\bar{M}(x)=E \bar{I}(x) W^{\prime \prime}(x)=0, \bar{Q}(x)=-E \bar{I}(x) W^{\prime \prime \prime}(x)$ $=0$ at free end

(ii) $W(x)=0, W^{\prime}(x)=0$ at clamped end

(iii) $W(x)=0, \bar{M}(x)=E \bar{I}(x) W^{\prime \prime}(x)=0$ at pinned end

For the F-F STB shown in Fig. 1, from Eqs. $(16 a, b)$ one has

$W^{\prime \prime}\left(L_{0}\right)=0, \quad W^{\prime \prime \prime}\left(L_{0}\right)=0$

$W^{\prime \prime}\left(L_{1}\right)=0, \quad W^{\prime \prime \prime}\left(L_{1}\right)=0$

Substituting Eq. (15) into Eqs. (19a,b) and $(20 \mathrm{a}, \mathrm{b})$ produces [28].

$c_{1} J_{3}\left(z_{0}\right)+c_{2} Y_{3}\left(z_{0}\right)+c_{3} I_{3}\left(z_{0}\right)+c_{4} K_{3}\left(z_{0}\right)=0$

$c_{1} J_{4}\left(z_{0}\right)+c_{2} Y_{4}\left(z_{0}\right)-c_{3} I_{4}\left(z_{0}\right)+c_{4} K_{4}\left(z_{0}\right)=0$

$c_{1} J_{3}\left(z_{1}\right)+c_{2} Y_{3}\left(z_{1}\right)+c_{3} I_{3}\left(z_{1}\right)+c_{4} K_{3}\left(z_{1}\right)=0$

$c_{1} J_{4}\left(z_{1}\right)+c_{2} Y_{4}\left(z_{1}\right)-c_{3} I_{4}\left(z_{1}\right)+c_{4} K_{4}\left(z_{1}\right)=0$

where 
$z_{0}=2 \beta L_{1} \xi_{0}^{1 / 2}, \quad z_{1}=2 \beta L_{1} \xi_{1}^{1 / 2}=2 \beta L_{1}, \quad \xi_{0}=L_{0} / L_{1}$

$(22 \mathrm{a}, \mathrm{b}, \mathrm{c})$

The non-trivial solution of Eqs. $(21 a-d)$ requires that

$\left|\begin{array}{llll}J_{3}\left(z_{0}\right) & Y_{3}\left(z_{0}\right) & I_{3}\left(z_{0}\right) & K_{3}\left(z_{0}\right) \\ J_{4}\left(z_{0}\right) & Y_{4}\left(z_{0}\right) & -I_{4}\left(z_{0}\right) & K_{4}\left(z_{0}\right) \\ J_{3}\left(z_{1}\right) & Y_{3}\left(z_{1}\right) & I_{3}\left(z_{1}\right) & K_{3}\left(z_{1}\right) \\ J_{4}\left(z_{1}\right) & Y_{4}\left(z_{1}\right) & -I_{4}\left(z_{1}\right) & K_{4}\left(z_{1}\right)\end{array}\right|=0$

which is the frequency equation for the F-F STB. From this equation, the values of $\beta=\beta_{r}$ $(r=1,2,3, \ldots)$ can be obtained using the half-interval method [11,28], while the corresponding natural frequencies can be obtained directly from Eq. (9) as

$\omega_{r}=\beta_{r}^{2} \sqrt{E \bar{I}_{1} /\left(\rho A_{1}\right)} \quad(r=1,2,3, \ldots)$

Finally, the corresponding mode shapes are determined by Eq. (15) as

$W_{r}(x)=x^{-1 / 2}\left[c_{1} J_{1}\left(z_{r}\right)+c_{2} Y_{1}\left(z_{r}\right)+c_{3} I_{1}\left(z_{r}\right)+c_{4} K_{1}\left(z_{r}\right)\right]$

or

$W_{r}(\xi)=L_{1}^{-1 / 2} \xi^{-1 / 2}\left[c_{1} J_{1}\left(z_{r}\right)+c_{2} Y_{1}\left(z_{r}\right)+c_{3} I_{1}\left(z_{r}\right)+c_{4} K_{1}\left(z_{r}\right)\right]$

where

$z_{r}=2 \beta_{r} L_{1} \xi^{1 / 2}=2 \beta_{r} L_{1}\left(x / L_{1}\right)^{1 / 2}$

The mode shapes $W_{r}(\xi)$ given by Eq. (25b) are the natural mode shapes. However, MSM requires the normal mode shapes rather than the natural ones and hence the former mode shapes must first be determined using the following procedure.

The orthonormality conditions for the normal mode shapes are given by [28] as

$\int_{\xi_{0}}^{1} \tilde{W}_{r}(\xi) \cdot \rho A(\xi) \cdot \tilde{W}_{s}(\xi) \cdot L_{1} d \xi=\delta_{r s}$

where $\delta_{r s}$ is the Kronecker's delta.

For convenience, one sets

$\widetilde{W}_{r}(\xi)=\widetilde{A}_{r} W_{r}(\xi)$

Substituting Eqs. (28) and (11) into Eq. (27), with $s=r$, produces

$\widetilde{\Delta}_{r}=\tilde{A}_{r}^{2} \int_{\xi_{0}}^{1} W_{r}(\xi) \cdot \rho A_{1} \xi \cdot W_{r}(\xi) \cdot L_{1} d \xi=1$

Introducing Eq. (25b) into Eq. (29) leads to
$\widetilde{\Delta}_{r}=\widetilde{A}_{r}^{2} \rho A_{1} \widetilde{B}_{r}=1$

where

$\widetilde{B}_{r}=\int_{\xi_{0}}^{1}\left[c_{1 r} J_{1}\left(z_{r}\right)+c_{2 r} Y_{1}\left(z_{r}\right)+c_{3 r} I_{1}\left(z_{r}\right)+c_{4 r} K_{1}\left(z_{r}\right)\right]^{2} d \xi$

From Eq. (30) one obtains

$\widetilde{A}_{r}=1 / \sqrt{\rho A_{1} \widetilde{B}_{r}}$

Finally, from Eq. (28), one obtains the $r$ th normal mode shape as

$\widetilde{W}_{r}(\xi)=\widetilde{A}_{r} W_{r}(\xi)=W_{r}(\xi) / \sqrt{\rho A_{1} \widetilde{B}_{r}}$

The analytical integration of Eq. (31) is difficult. Thus, in the present study, the values of $B_{r}(r=1,2,3, \ldots)$ are obtained from the numerical integrations using Simpson's rule [10,27]; and [30]. It is noted that the integration is performed from $x=L_{0}$ to $x=L_{1}$ (or $\xi=\xi_{0}$ to $\xi=1.0$ ) with $\xi=x / L_{1}$ and $\xi_{0}=L_{0} / L_{1}$ (see Fig. 1).

\subsection{Free rigid-body motions of F-F bare STB}

This subsection derives the natural frequencies and associated normal mode shapes of the considered F-F bare STB during free rigid-body motion. The location of the center of gravity (c.g.) of the bare STB, $C$, is determined as follows (see Figs. 1 and 2):

The total mass of the STB is given by

$m_{\text {ref }}=\rho b_{0} \frac{1}{2}\left(h_{0}+h_{1}\right)\left(L_{1}-L_{0}\right)=\frac{1}{2} \rho A_{1} L_{1}\left(1+\xi_{0}\right)\left(1-\xi_{0}\right)$

Furthermore, the mass moment of the STB about the origin $o$ is given by

$$
\begin{aligned}
m_{\mathrm{ref}} x_{C} & =\int_{L_{0}}^{L_{1}} \rho b_{0} h(x) x d x=\rho b_{0} \int_{\xi_{0}}^{1}\left(h_{1} \xi\right) \cdot L_{1} \xi \cdot L_{1} d \xi \\
& =\frac{1}{3} \rho A_{1} L_{1}^{2}\left(1-\xi_{0}^{3}\right)
\end{aligned}
$$

Thus, from Eqs. (34) and (35), one obtains the axial coordinate for the c.g. of the STB as

$x_{C}=\frac{2}{3} L_{1}\left(\frac{1+\xi_{0}+\xi_{0}^{2}}{1+\xi_{0}}\right)$

The distance of the c.g. $(C)$ from the large end of the STB is given as (see Fig. 1) 


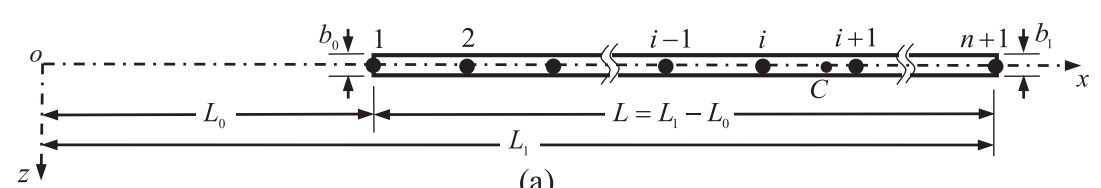

(a)

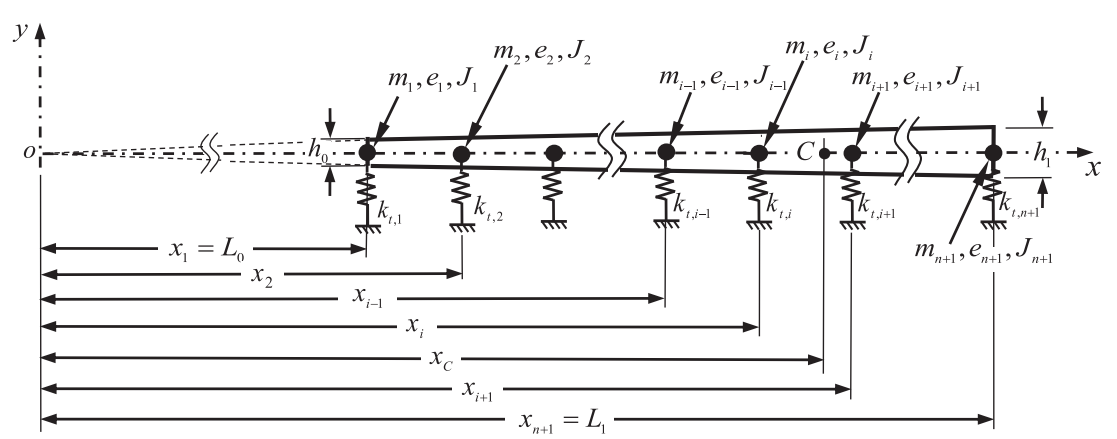

(b)

Fig. 2. Schematic illustration of free-free (F-F) STB carrying $n+1$ sets of concentrated elements (CEs) with each set of CEs consisting of a lumped mass $m_{i}$ (with eccentricity $e_{i}$ and rotary inertia $J_{i}$ ) and a translational spring with stiffness $k_{t, i}$ : (a) top view; (b) front view.

$f=L_{1}-x_{C}=\frac{1}{3} L_{1}\left(\frac{1+\xi_{0}-2 \xi_{0}^{2}}{1+\xi_{0}}\right)$

From [31,32]; the two natural mode shapes for the rigid-body (heave and pitch) motions of the F-F bare STB are obtained as

$$
\begin{gathered}
W_{1}(x)=C_{1} \text { (heave), } W_{2}(x)=C_{2}\left(x-x_{C}\right) \text { (pitch) } \\
\left(\text { for } L_{0} \leq x \leq L_{1}\right)
\end{gathered}
$$

or

$$
W_{1}(\xi)=C_{1} \text { (heave), } W_{2}(\xi)=L_{1} C_{2}\left(\xi-\xi_{C}\right) \text { (pitch) }
$$$$
\text { (for } \xi_{0} \leq \xi \leq 1.0 \text { ) }
$$

where

$\xi=x / L_{1}, \xi_{C}=x_{C} / L_{1}, \xi_{0}=L_{0} / L_{1}$

According to the $x y z$ coordinate system shown in Fig. 1, Eq. (38a) represents the translation of the entire F-F STB in the transverse $y$-direction, while Eq. (38b) represents the rotation of the entire F-F STB about its c.g. $(C)$.

In order to determine the associated normalization factors, one sets (see Eqs. (27) and (38a,b))

$\widetilde{W}_{1}(x)=\widetilde{C}_{1}, \widetilde{W}_{2}(x)=\widetilde{C}_{2}\left(x-x_{C}\right)$
$\int_{L_{0}}^{L_{1}} \rho A(x) \tilde{W}_{r}^{2}(x) d x=1 \quad($ for $r=1,2, \ldots)$

Substituting Eqs. (11) and (41a) into Eq. (41c) for $r=1$ yields

$$
\begin{aligned}
& \int_{L_{0}}^{L_{1}} \rho A(x) \widetilde{W}_{1}^{2}(x) d x=\int_{\xi_{0}}^{1} \rho A_{1} \xi \widetilde{C}_{1}^{2} \cdot L_{1} d \xi \\
& =\frac{1}{2} \rho A_{1} L_{1}\left(1-\xi_{0}^{2}\right) \widetilde{C}_{1}^{2}=1
\end{aligned}
$$

Thus, the normalization factor for the first rigid-body mode shape is given by

$$
\widetilde{C}_{1}=1 / \sqrt{\frac{1}{2} \rho A_{1} L_{1}\left(1-\xi_{0}^{2}\right)} \quad(\text { heave })
$$

Similarly, substituting Eqs. (11) and (41b) into Eq. (41c) for $r=2$ gives

$$
\begin{gathered}
\int_{L_{0}}^{L_{1}} \rho A(x) \tilde{W}_{2}^{2}(x) d x=\frac{1}{L_{1}} \rho A_{1} \widetilde{C}_{2}^{2} \int_{L_{0}}^{L_{1}} x\left(x-x_{C}\right)^{2} d x \\
=\rho A_{1} L_{1}^{3} C_{2}^{2} \cdot\left[\frac{1}{4}\left(1-\xi_{0}^{4}\right)-\frac{2}{3} \xi_{C}\left(1-\xi_{0}^{3}\right)+\frac{1}{2} \xi_{C}^{2}\left(1-\xi_{0}^{2}\right)\right]=1
\end{gathered}
$$


Thus, the normalization factor for the second rigid-body mode shape has the form

$$
\widetilde{C}_{2}=\frac{1}{\sqrt{\rho A_{1} L_{1}^{3}\left[\frac{1}{4}\left(1-\xi_{0}^{4}\right)-\frac{2}{3} \xi_{C}\left(1-\xi_{0}^{3}\right)+\frac{1}{2} \xi_{C}^{2}\left(1-\xi_{0}^{2}\right)\right]}}(\text { pitch })
$$

From Eqs. $(41 a, b),(42 b)$ and $(43 b)$, one obtains the normal mode shapes and their derivations for the rigid-body motions of the F-F bare STB as

$$
\begin{aligned}
& \widetilde{W}_{R 1}(x)=\widetilde{C}_{1}, \widetilde{W}_{R 1}^{\prime}(x)=\widetilde{W}_{R 1}^{\prime \prime}(x)=0 \text { (heave) } \\
& \quad\left(\text { for } L_{0} \leq x \leq L_{1}\right) \\
& \quad(44 \mathrm{a}, \mathrm{b}, \mathrm{c}) \\
& \tilde{W}_{R 2}(x)=\widetilde{C}_{2}\left(x-x_{C}\right), \widetilde{W}_{R 2}^{\prime}(x)=\widetilde{C}_{2}, \widetilde{W}_{R 2}^{\prime \prime}(x)=0(\text { pitch }) \\
& \quad\left(\text { for } L_{0} \leq x \leq L_{1}\right)
\end{aligned}
$$

or

$$
\begin{aligned}
& \widetilde{W}_{R 1}(\xi)=\widetilde{C}_{1}, \widetilde{W}_{R 1}^{\prime}(\xi)=\widetilde{W}_{R 1}^{\prime \prime}(\xi)=0 \text { (heave) } \\
& \quad\left(\text { for } \xi_{0} \leq \xi \leq 1.0\right)
\end{aligned}
$$$$
\begin{gathered}
\widetilde{W}_{R 2}(\xi)=L_{1} \widetilde{C}_{2}\left(\xi-\xi_{C}\right), \widetilde{W}_{R 2}^{\prime}(\xi)=L_{1} \widetilde{C}_{2}, \widetilde{W}_{R 2}^{\prime \prime}(\xi)=0 \\
\text { (pitch) } \quad\left(\text { for } \xi_{0} \leq \xi \leq 1.0\right)
\end{gathered}
$$

The corresponding natural frequencies are

$\omega_{R 1}=\omega_{R 2}=0$

Note that in Eqs. (44)-(48), the subscript " $R$ " denotes rigid-body motion of the beam.

\subsection{Free elastic vibration coupled with rigid-body motions of F-F loaded STB}

This subsection determines the natural frequencies and mode shapes of coupled rigid-body motion and elastic vibrations of the F-F (loaded) STB carrying various CEs. As shown in Fig. 2, the F-F STB is assumed to carry $n+1$ sets of CEs, where each set of CEs consists of a lumped mass $m_{i}$ (possessing eccentricity $e_{i}$ and rotary inertia $J_{i}$ ) and a translational spring with stiffness $k_{t, i}$. From Eq. (1) of this paper and Eq. (35) of [31]; the equation of motion of the F-F loaded STB, with $u_{y} \equiv u_{y}(x, t)$, is obtained as

$$
\begin{aligned}
& \frac{\partial^{2}}{\partial x^{2}}\left[E \bar{I}(x) \frac{\partial^{2} u_{y}}{\partial x^{2}}\right]+\rho A(x) \frac{\partial^{2} u_{y}}{\partial t^{2}} \\
& =\sum_{i=1}^{n+1}\left\{-\left(m_{i} \ddot{u}_{y}+k_{t, i} u_{y}\right)\right. \\
& \left.+\left(J_{i}+m_{i} e_{i}^{2}\right) \ddot{u}_{y}^{\prime \prime}\right\} \delta\left(x-x_{i}\right)
\end{aligned}
$$

where $\delta(\cdot)$ denotes the Dirac delta function and the other symbols are as defined previously for Eq. (1).

According to the mode-superposition method (MSM) and the expansion theorem of [6,23], one may set

$u_{y}(x, t)=\sum_{s=1}^{n^{\prime}} \tilde{W}_{s}(x) \eta_{s}(t)$

where $\tilde{W}_{s}(x)$ is the $s$-th normal mode shape of the F$\mathrm{F}$ bare STB, $\eta_{s}(t)$ is the associated generalized coordinate, and $n^{\prime}$ is the total number of modes considered.

Substituting Eq. (50) into Eq. (49), pre-multiplying the resulting expression by $\sum_{r=1}^{n^{\prime}} W_{r}(x) d x$, and then integrating each term over the whole length of the STB (i.e., $x=L_{0}$ to $L_{1}$ ) gives

$$
\begin{gathered}
\sum_{r=1}^{n^{\prime}} \sum_{s=1}^{n^{\prime}} \int_{L_{0}}^{L_{1}} \tilde{W}_{r}(x) \cdot \frac{d^{2}}{d x^{2}}\left[E \bar{I}(x) \frac{d^{2} \tilde{W}_{s}(x)}{d x^{2}}\right] d x \cdot \eta_{s}(t) \\
+\sum_{r=1}^{n^{\prime}} \sum_{s=1}^{n^{\prime}} \int_{L_{0}}^{L_{1}} \tilde{W}_{r}(x) \cdot \rho A(x) \tilde{W}_{s} d x \cdot \ddot{\eta}_{s}(t) \\
=\sum_{i=1}^{n+1} \sum_{r=1}^{n^{\prime}} \sum_{s=1}^{n^{\prime}} \int_{L_{0}}^{L_{1}} \tilde{W}_{r} \cdot\left\{\left[-m_{i} \tilde{W}_{s}+\left(J_{i}+m_{i} e_{i}^{2}\right)\right.\right. \\
\left.\left.\times \tilde{W}_{s}^{\prime \prime}\right] \cdot \ddot{\eta}_{s}(t)-k_{t, i} \tilde{W}_{s} \cdot \eta_{s}(t)\right\} d x \cdot \delta\left(x-x_{i}\right)
\end{gathered}
$$

For free vibrations, the transverse displacement term $u_{y}(x, t)$ in Eq. (1) may take the form

$u_{y}(x, t)=\tilde{W}_{s}(x) e^{j \omega_{s} t}$

where $\tilde{W}_{s}(x)$ is the s-th normal mode shape and $\omega_{s}$ is the corresponding $s$-th natural frequency of the bare STB.

From Eqs. (1) and (52), one obtains

$$
\frac{d^{2}}{d x^{2}}\left[E \bar{I}(x) \frac{d^{2} \tilde{W}_{s}(x)}{d x^{2}}\right]-\rho A(x) \omega_{s}^{2} \tilde{W}_{s}(x)=0
$$


Pre-multiplying Eq. (53) by $\tilde{W}_{r}(x) d x$, integrating each term over the whole beam length (from $x=L_{0}$ to $L_{1}$ ) and then introducing the orthonormality conditions for normal mode shapes, one has

$$
\begin{aligned}
& \int_{L_{0}}^{L_{1}} \tilde{W}_{r}(x) \frac{d^{2}}{d x^{2}}\left[E \bar{I}(x) \frac{d^{2} \tilde{W}_{s}(x)}{d x^{2}}\right] d x \\
& =\int_{L_{0}}^{L_{1}} \widetilde{W}_{r}(x) \rho A(x) \omega_{s}^{2} \widetilde{W}_{s}(x) d x=\omega_{s}^{2} \delta_{r s}
\end{aligned}
$$

It is noted that, according to Eq. (27), one has

$$
\int_{L_{0}}^{L_{1}} \tilde{W}_{r}(x) \rho A(x) \tilde{W}_{s}(x) d x=\delta_{r s}
$$

Introducing the relationships given in Eqs. (54) and (55) into Eq. (51) yields

$$
\begin{aligned}
\sum_{r=1}^{n^{\prime}} \sum_{s=1}^{n^{\prime}} \omega_{s}^{2} \delta_{r s} \cdot \eta_{s}(t)+\sum_{r=1}^{n^{\prime}} \sum_{s=1}^{n^{\prime}} \delta_{r s} \cdot \ddot{\eta}_{s}(t) \\
\quad=\sum_{i=1}^{n+1} \sum_{r=1}^{n^{\prime}} \sum_{s=1}^{n^{\prime}} \int_{L_{0}}^{L_{1}} \tilde{W}_{r} \cdot\left\{\left[-m_{i} \widetilde{W}_{s}\right.\right. \\
\left.\left.\quad+\left(J_{i}+m_{i} e_{i}^{2}\right) \tilde{W}_{s}^{\prime \prime}\right] \cdot \ddot{\eta}_{s}(t)-k_{t, i} \tilde{W}_{s} \cdot \eta_{s}(t)\right\} d x \cdot \delta\left(x-x_{i}\right)
\end{aligned}
$$

or

$$
\begin{aligned}
& \left(\left[\omega^{2} \backslash\right]_{n^{\prime} \times n^{\prime}}+\sum_{i=1}^{n+1} k_{t, i}\left[S_{r s}^{W W}\left(x_{i}\right)\right]_{n^{\prime} \times n^{\prime}}\right)\{\eta(t)\}_{n^{\prime} \times 1} \\
& +\left(\left[{ }^{\backslash} \backslash\right]_{n^{\prime} \times n^{\prime}}+\sum_{i=1}^{n+1} m_{i}\left[S_{r s}^{W W}\left(x_{i}\right)\right]_{n^{\prime} \times n^{\prime}}\right. \\
& \left.-\sum_{i=1}^{n+1}\left(J_{i}+m_{i} e_{i}^{2}\right)\left[S_{r s}^{W W^{\prime \prime}}\left(x_{i}\right)\right]_{n^{\prime} \times n^{\prime}}\right)\{\ddot{\eta}(t)\}_{n^{\prime} \times 1}=0
\end{aligned}
$$

where the symbols $\left[{ }^{\backslash} \omega^{2} \backslash\right]$ and $\left[{ }^{\backslash} I \backslash\right]$ denote a diagonal matrix and an identity matrix, respectively, while the other symbols are defined as follows:

$$
S_{r s}^{W W}\left(x_{i}\right)=\int_{L_{0}}^{L_{1}} \tilde{W}_{r}(x) \tilde{W}_{s}(x) d x \cdot \delta\left(x-x_{i}\right) \equiv \tilde{W}_{r}\left(x_{i}\right) \tilde{W}_{s}\left(x_{i}\right)
$$

$$
S_{r s}^{W W^{\prime \prime}}\left(x_{i}\right)=\int_{L_{0}}^{L_{1}} \tilde{W}_{r}(x) \tilde{W}_{s}^{\prime \prime}(x) d x \cdot \delta\left(x-x_{i}\right) \equiv \tilde{W}_{r}\left(x_{i}\right) \tilde{W}_{s}^{\prime \prime}\left(x_{i}\right)
$$

$$
\begin{aligned}
& {\left[S_{r s}^{W V}\left(x_{i}\right)\right]_{n^{\prime} \times n^{\prime}}} \\
& =\left[\begin{array}{llll}
\tilde{W}_{1}\left(x_{i}\right) V_{1}\left(x_{i}\right) & \tilde{W}_{1}\left(x_{i}\right) V_{2}\left(x_{i}\right) & \cdots & \tilde{W}_{1}\left(x_{i}\right) V_{n^{\prime}}\left(x_{i}\right) \\
\tilde{W}_{2}\left(x_{i}\right) V_{1}\left(x_{i}\right) & \tilde{W}_{2}\left(x_{i}\right) V_{2}\left(x_{i}\right) & \cdots & \tilde{W}_{2}\left(x_{i}\right) V_{n^{\prime}}\left(x_{i}\right) \\
\vdots & \vdots & \ddots & \vdots \\
\tilde{W}_{n^{\prime}}\left(x_{i}\right) V_{1}\left(x_{i}\right) & \tilde{W}_{n^{\prime}}\left(x_{i}\right) V_{2}\left(x_{i}\right) & \cdots & \tilde{W}_{n^{\prime}}\left(x_{i}\right) V_{n^{\prime}}\left(x_{i}\right)
\end{array}\right] \\
& \text { (for } \left.V=\tilde{W} \text { or } \tilde{W}^{\prime \prime}\right)
\end{aligned}
$$

For convenience, let Eq. (56b) be rewritten as

$$
[m]_{n^{\prime} \times n^{\prime}}\{\ddot{\eta}(t)\}_{n^{\prime} \times 1}+[k]_{n^{\prime} \times n^{\prime}}\{\eta(t)\}_{n^{\prime} \times 1}=0
$$

where

$$
\begin{aligned}
{[m]_{n^{\prime} \times n^{\prime}}=} & {\left[I_{\backslash}\right]_{n^{\prime} \times n^{\prime}}+\sum_{i=1}^{n+1} m_{i}\left[S_{r s}^{W W}\left(x_{i}\right)\right]_{n^{\prime} \times n^{\prime}} } \\
& -\sum_{i=1}^{n+1}\left(J_{i}+m_{i} e_{i}^{2}\right)\left[S_{r s}^{W W}\left(x_{i}\right)\right]_{n^{\prime} \times n^{\prime}} \\
{[k]_{n^{\prime} \times n^{\prime}}=} & {\left[{ }^{\backslash} \omega^{2}\right]_{n^{\prime} \times n^{\prime}}+\sum_{i=1}^{n+1} k_{t, i}\left[S_{r s}^{W W}\left(x_{i}\right)\right]_{n^{\prime} \times n^{\prime}} }
\end{aligned}
$$

For the presented MMSM, the lowest two natural frequencies in Eq. (59b) are equal to those for the rigid-body motions of the F-F bare STB, i.e., $\omega_{1}=\omega_{R 1}=0$ and $\omega_{2}=\omega_{R 2}=0$. Meanwhile, the other natural frequencies are equal to those for the elastic vibrations of the F-F bare STB.

For free vibrations of the F-F loaded beam (carrying various $\mathrm{CEs}$ ), one has

$\{\eta(t)\}=\{\bar{\eta}\} e^{j \bar{\omega} t}$

where $\{\bar{\eta}\}$ is the amplitude of $\{\eta(t)\}, \bar{\omega}$ is the natural frequency of the F-F loaded STB, $t$ is time, and $j=$ $\sqrt{-1}$.

Substituting Eq. (60) into Eq. (58) gives

$$
\left([k]-\bar{\omega}^{2}[m]\right)\{\bar{\eta}\}=0
$$

or

$$
[k]\{\bar{\eta}\}=[m]\{\bar{\eta}\}\left[{ }^{\backslash} \bar{\omega}^{2} \backslash\right]
$$

which is the characteristic equation. From Eq. (61b), the natural frequencies $\bar{\omega}_{r}\left(r=1 \sim n^{\prime}\right)$ and associated generalized coordinate vectors $\{\bar{\eta}\}_{r}\left(r=1 \sim n^{\prime}\right)$ can be obtained using the Jacobi method $[5,28]$. Meanwhile, the $r$-th mode shape of the F-F loaded STB can be determined by

$\bar{W}_{r}(x)=[\tilde{W}(x)]\{\bar{\eta}\}_{r} \quad\left(r=1 \sim n^{\prime}\right)$ 
(a)

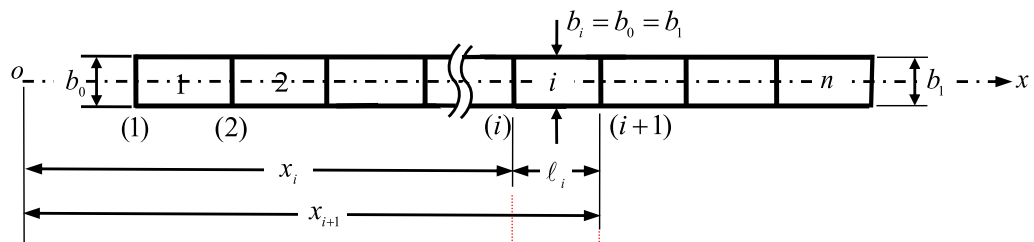

(b)

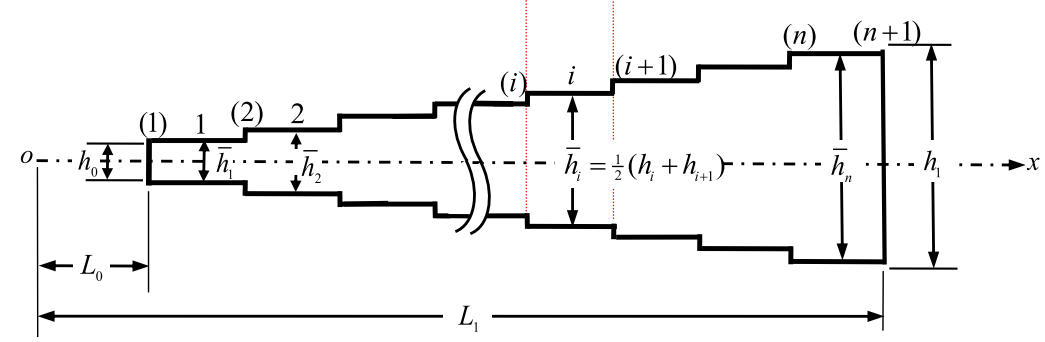

Fig. 3. Finite element model of free-free (F-F) single-tapered beam composed of $n$ beam elements: (a) top view; (b) front view. Note that (1), (2), $\ldots,(i),(i+1), \ldots$ represent the numberings of nodes and $1,2, \ldots, i, i+1, \ldots$ represent the numberings of beam elements.

where

$$
\begin{aligned}
& {\left[\begin{array}{l}
\widetilde{W}(x)
\end{array}\right]=\left[\begin{array}{llll}
\widetilde{W}_{1}(x) & \widetilde{W}_{2}(x) & \cdots & \widetilde{W}_{n^{\prime}}(x)
\end{array}\right]} \\
& \{\bar{\eta}\}_{r}=\left[\begin{array}{llll}
\bar{\eta}_{1} & \bar{\eta}_{2} & \cdots & \bar{\eta}_{n^{\prime}}
\end{array}\right]_{r}^{T}
\end{aligned}
$$

For the presented MMSM, the lowest two normal mode shapes in Eq. (63a) are equal to those for the rigid-body motions of the $\mathrm{F}_{\sim} \mathrm{F}$ bare STB, i.e., $W_{1}(x)=W_{R 1}(x)$ and $W_{2}(x)=W_{R 2}(x)$, while the other mode shapes are equal to those for the elastic vibrations of the F-F bare STB. It is noted that the order of Eq. (61a) or (61b) derived from MMSM, i.e., $n^{\prime}$, is much smaller than that of Eq. (74) derived from FEM (see Section 3), i.e., $2(n+1)$. Consequently, the CPU time required by MMSM is much smaller than that of FEM, and hence MMSM is better suited to the development of real-time-demonstration technology.

\section{Free vibration analysis of F-F single-tapered beam by FEM}

In order to determine the natural frequencies and associated mode shapes of the bare STB or the loaded STB with FEM, one must firstly replace the tapered beam shown in Fig. 1 or 2 by an equivalent multi-step non-uniform beam composed of multiple uniform beam elements, as shown in Fig. 3. According to Eqs. (6) and (10), the height $h_{i}$ and area $A_{i}$ of the cross-section located at node $i$ are given respectively by

$h_{i}=\xi_{i} h_{1}, A_{i}=b_{i} h_{i}$

where

$\xi_{i}=x_{i} / L_{1}$
Thus, the average height $\bar{h}_{i}$, average area $\bar{A}_{i}$, and average moment of inertia $\bar{I}_{i}$ of the $i$-th beam element between nodes $i$ and $i+1$ are determined respectively by

$\bar{h}_{i}=\left(h_{i}+h_{i+1}\right) / 2, \bar{A}_{i}=b_{i} \bar{h}_{i}, \bar{I}_{i}=b_{i} \bar{h}_{i}^{3} / 12 \quad(69 \mathrm{a}, \mathrm{b}, \mathrm{c})$

Based on the average parameters given in Eqs. $(69 a-c)$ and the material constants (mass density $\rho$ and Young's modulus $E$ ) of the beam, the stiffness matrix $[k]_{i}$ and mass matrix $[m]_{i}$ of the $i$-th bare (uniform) beam element are obtained respectively as

$\begin{aligned} {[k]_{i}=} & {\left[\begin{array}{llll}k_{11} & k_{12} & k_{13} & k_{14} \\ k_{21} & k_{22} & k_{23} & k_{24} \\ k_{31} & k_{32} & k_{33} & k_{34} \\ k_{41} & k_{42} & k_{43} & k_{44}\end{array}\right], } \\ {[m]_{i}=} & {\left[\begin{array}{llll}m_{11} & m_{12} & m_{13} & m_{14} \\ m_{21} & m_{22} & m_{23} & m_{24} \\ m_{31} & m_{32} & m_{33} & m_{34} \\ m_{41} & m_{42} & m_{43} & m_{44}\end{array}\right] }\end{aligned}$

Meanwhile, the corresponding matrices for the $i$-th loaded beam element are given as

$[\bar{k}]_{i}=\left[\begin{array}{llll}k_{11}+k_{t, i} & k_{12} & k_{13} & k_{14} \\ k_{21} & k_{22} & k_{23} & k_{24} \\ k_{31} & k_{32} & k_{33}+k_{t, i+1} & k_{34} \\ k_{41} & k_{42} & k_{43} & k_{44}\end{array}\right]$

The coefficients $k_{i j}$ and $m_{i j}(i, j=1-4)$ in the stiffness matrices, $[k]_{i}$ and $[\bar{k}]_{i}$, and mass matrices, 
$[\bar{m}]_{i}=\left[\begin{array}{llll}m_{11}+m_{i} & m_{12}+e_{i} m_{i} & m_{13} & m_{14} \\ m_{21}+e_{i} m_{i} & m_{22}+J_{i}+e_{i}^{2} m_{i} & m_{23} & m_{24} \\ m_{31} & m_{32} & m_{33}+m_{i+1} & m_{34}+e_{i+1} m_{i+1} \\ m_{41} & m_{42} & m_{43}+e_{i+1} m_{i+1} & m_{44}+J_{i+1}+e_{i+1}^{2} m_{i+1}\end{array}\right]$

$[m]_{i}$ and $[\bar{m}]_{i}$, of the bare beam element in Eqs. $(70 a, b)$ and $(71 a, b)$ can be obtained from the existing literature [24,28]; and [29], while the additional terms (such as $k_{t, j}, m_{j}, J_{j}+e_{j}^{2} m_{j}$ and $e_{j} m_{j}$, with $j=i$, $i+1)$ appearing in Eqs. $(71 \mathrm{a}, \mathrm{b})$ for the loaded beam element reflect the contributions of the CEs to the relevant stiffnesses, masses and mass moments of inertia, and can be obtained from [28].

Once the property matrices for each beam element have been determined from Eqs. (70) or (71), the general assembly method can be used to obtain the overall stiffness matrix $[K]$ and overall mass matrix $[M]$ for the entire bare beam or loaded beam. The equation of motion for the entire vibrating system is then given by

$[M]\{\ddot{u}(t)\}+[K]\{u(t)\}=0$

For a F-F beam such as that shown in Figs. 1 or 2, no constrained DOF must be eliminated. Thus, assuming that the entire beam is composed of $n_{e}(=$ $n$ ) beam elements, the order of $[K]$ or $[M]$ in Eq. (72) is $2\left(n_{e}+1\right) \times 2\left(n_{e}+1\right)$.
For free vibrations, one has

$$
\{u(t)\}=\{U\} e^{j \omega t}
$$

where $\{U\}$ denotes the amplitude of the nodedisplacement vector $\{u(t)\}, \omega$ is the natural frequency of the bare beam or loaded beam (and is dependent on the property matrices $[K]$ and $[M]), t$ is the time, and $j=\sqrt{-1}$. Substituting Eq. (73) into Eq. (72) leads to

$$
[K][U]=[M][U]\left[{ }^{`} \omega^{2} \backslash\right]
$$

where

$$
\begin{aligned}
& {[U]_{2\left(n_{e}+1\right) \times 2\left(n_{e}+1\right)}=\left\lceil\{U\}_{1}\{U\}_{2} \cdots\{U\}\right\rfloor_{2\left(n_{e}+1\right)}} \\
& {\left[{ }^{\top} \omega^{2} \backslash\right]_{2\left(n_{e}+1\right) \times 2\left(n_{e}+1\right)}=\left\lceil\omega_{1}^{2} \omega_{2}^{2} \cdots \omega_{2\left(n_{e}+1\right)}^{2}\right\rfloor_{2\left(n_{e}+1\right) \times 2\left(n_{e}+1\right)}}
\end{aligned}
$$

in which the symbol \lceil\rfloor denoting a diagonal matrix. Eq. (74) is a standard eigenproblem equation, and hence the natural frequencies $\omega_{r}$ and the associated

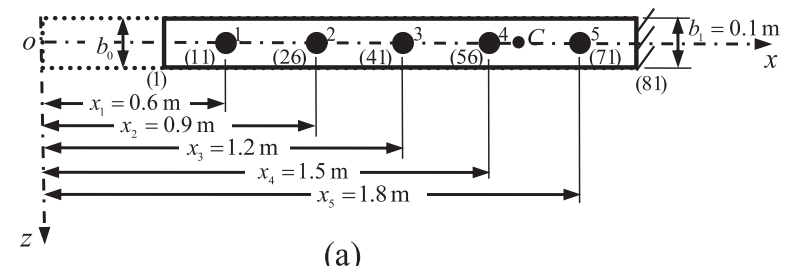

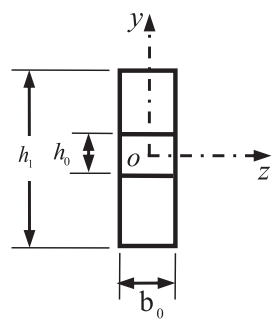

(c)

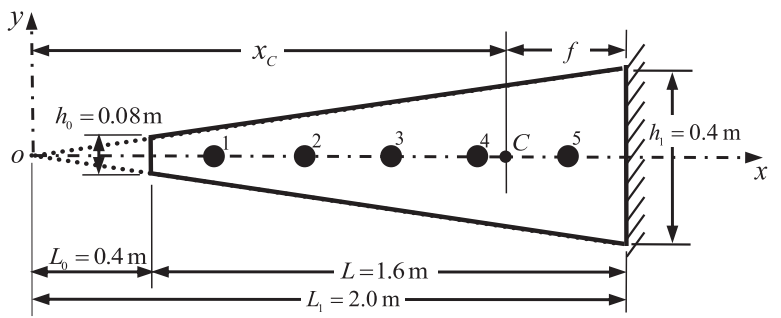

(b)

Fig. 4. Truncated F-C STB (with $\rho=7850 \mathrm{~kg} / \mathrm{m}^{3}$ and $E=2.051 \times 10^{11} \mathrm{~N} / \mathrm{m}^{2}$ ) carrying five identical lumped masses (each with $m_{i}^{*}=m_{i} / m_{r e f}=$ 0.2 ) at $x_{1}=0.6 m$ (node 11), $x_{2}=0.9 m$ (node 26 ), $x_{3}=1.2 m$ (node 41 ), $x_{4}=1.5 m$ (node 56) and $x_{5}=1.8 m$ (node 71): (a) top view; (b) front view; (c) left side view. 
Table 1. Lowest several natural frequencies of truncated F-C STB (Fig. 4) obtained from MMSM (with $n^{\prime}=6$ and $n_{\text {Simpson }}=400$ ) and FEM (with $n_{e}=80$ ) for: (a) bare beam (no attachments), (b) loaded beam (carrying five identical lumped masses each with $m_{i}^{*}=m_{i} / m_{r e f}=0.2$ ).

\begin{tabular}{|c|c|c|c|c|c|c|c|}
\hline \multicolumn{8}{|c|}{ (a) Bare beam } \\
\hline \multirow[t]{2}{*}{ Methods } & \multicolumn{6}{|c|}{ Natural frequencies of bare STB, $\omega_{r}(\mathrm{rad} / \mathrm{sec})$} & \multirow[t]{2}{*}{ Remarks } \\
\hline & $\omega_{1}$ & $\omega_{2}$ & $\omega_{3}$ & $\omega_{4}$ & $\omega_{5}$ & $\omega_{6}$ & \\
\hline MMSM & 989.6626 & 3629.5821 & 8503.9741 & $15,704.6849$ & $25,267.5120$ & $37,202.5661$ & ${ }^{\mathrm{a}}$ Exact \\
\hline [35] & 989.6626 & 3629.5821 & 8503.9741 & $15,704.6849$ & $25,267.5120$ & - & Exact \\
\hline FEM & 989.5017 & 3628.6310 & 8501.3306 & $15,699.4684$ & $25,258.8576$ & $37,189.6462$ & ${ }^{\mathrm{b}} n_{e}=80$ \\
\hline \multicolumn{8}{|c|}{ (b) Loaded beam } \\
\hline \multirow[t]{2}{*}{ Methods } & \multicolumn{6}{|c|}{ Natural frequencies of loaded STB, $\bar{\omega}_{r}(\mathrm{rad} / \mathrm{sec})$} & \multirow[t]{2}{*}{ Remarks } \\
\hline & $\bar{\omega}_{1}$ & $\bar{\omega}_{2}$ & $\bar{\omega}$ & & $\bar{\omega}_{4}$ & $\bar{\omega}_{5}$ & \\
\hline MMSM & 613.2201 & 2525.5385 & & & $12,184.0385$ & $16,089.9902$ & ${ }^{\mathrm{c}} n^{\prime}=6$ \\
\hline [35] & 613.2201 & 2525.5381 & & & $12,184.0282$ & $16,089.9494$ & $n^{\prime}=6$ \\
\hline FEM & 613.1226 & 2524.3388 & & & $12,105.9353$ & $15,885.0954$ & $n_{e}=80$ \\
\hline
\end{tabular}

${ }^{a}$ Obtained from exact solution for bare STB.

${ }^{\mathrm{b}} n_{e}=$ Total number of beam elements in FEM.

c $n^{\prime}=$ Total number of modes considered in MMSM.

natural mode shapes $\{U\}_{r}, r=1,2,3, \ldots, 2\left(n_{e}+1\right)$ can be obtained using the Jacobi method $[5,28]$.

\section{Numerical examples and discussions}

\subsection{Comparison with existing literature and FEM}

This section considers the truncated F-C STB given by [35]. The dimensions and material constants of the beam are set as follows (see Fig. 4): widths $b_{0}=b_{1}=0.1 \mathrm{~m}$, depths $h_{0}=0.08 \mathrm{~m}$ and $h_{1}=$ $0.4 \mathrm{~m}$, lengths $L_{0}=0.4 \mathrm{~m}$ and $L_{1}=2.0 \mathrm{~m}$, mass density $\rho=7850 \mathrm{~kg} / \mathrm{m}^{3}$, and Young's modulus $E=$ $2.051 \times 10^{11} \mathrm{~N} / \mathrm{m}^{2}$. Table 1 (a) lists the lowest six natural frequencies of the bare STB, while Table 1(b) lists the lowest five ones of the loaded STB carrying five identical point masses, each with $m_{i}^{*}=m_{i} / m_{\text {ref }}=0.2(i=1-5)$, located at $x_{i}=0.6 \mathrm{~m}$ (node 11), $0.9 \mathrm{~m}$ (node 26), $1.2 \mathrm{~m}$ (node 41 ), $1.5 \mathrm{~m}$ (node 56) and $1.8 \mathrm{~m}$ (node 71 ), respectively. (Note that the reference mass is equal to the total mass of the STB, i.e., $m_{\text {ref }}=\rho A_{\text {ave }} L=301.44 \mathrm{~kg}$ ). It is seen that the lowest six natural frequencies of the bare STB, $\omega_{r}(r=1 \sim 6)$, obtained from MMSM are very close to those obtained from FEM based on $n_{e}=80$. A similar observation is noted for the natural frequencies of the loaded STB $\bar{\omega}_{r}(r=1 \sim 5)$ obtained from MMSM (based on $n^{\prime}=6$ and $n_{\text {Simpson }}=400$ ) and FEM (based on $n_{e}=80$ ), respectively. Furthermore, all of the above-mentioned frequencies $\left(\omega_{r}\right.$ and $\bar{\omega}_{r}$ ) are very close to the corresponding frequencies given by [35]. Overall, the results confirm that the presented MMSM can be applied to the free vibration analysis of the constrained (e.g., clamped-

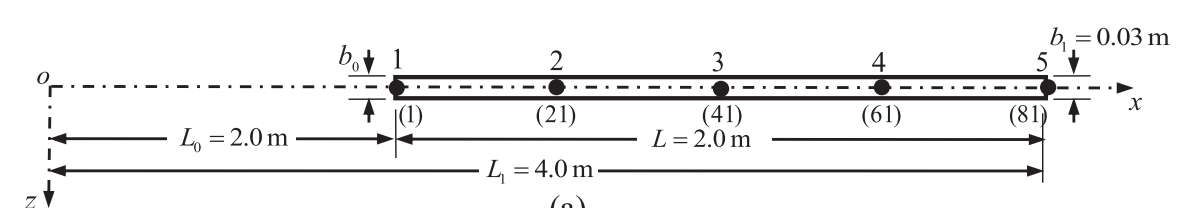

(a)

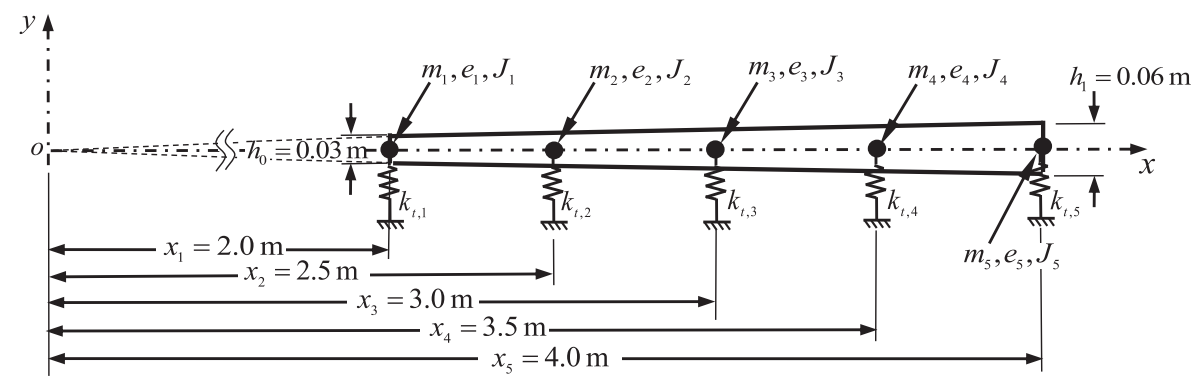

(b)

Fig. 5. Truncated F-F STB (with $\rho=7850 \mathrm{~kg} / \mathrm{m}^{3}$ and $E=2.068 \times 10^{11} \mathrm{~N} / \mathrm{m}^{2}$ ) carrying five sets of CEs at $x_{1}=2.0 \mathrm{~m}, x_{2}=2.5 \mathrm{~m}, x_{3}=3.0 \mathrm{~m}, x_{4}=$ $3.5 \mathrm{~m}$ and $x_{5}=4.0 \mathrm{~m}$ : (a) top view; (b) front view. 
Table 2. (a) Lowest six natural frequencies of F-F bare elastic STB (see Fig.5); (b) Effects of various CEs on lowest five natural frequencies of F-F loaded rigid-and-elastic-coupled STB carrying five sets of CEs (see Fig. 5) located at $x_{1}=2.0 \mathrm{~m}$ (node 1), $x_{2}=2.5 \mathrm{~m}$ (node 21), $x_{3}=3.0 \mathrm{~m}$ (node 41), $x_{4}=3.5 \mathrm{~m}$ (node 61) and $x_{5}=4.0 \mathrm{~m}$ (node 81), based on $n^{\prime}=6$ (or 8, including 2 rigid-body modes), $n_{\text {Simpson }}=400$ and $n_{e}=80$.

\begin{tabular}{|c|c|c|c|c|c|c|c|c|c|c|c|c|}
\hline \multicolumn{13}{|c|}{ (a) Bare elastic beam } \\
\hline \multirow{2}{*}{\multicolumn{4}{|c|}{ Methods }} & \multicolumn{8}{|c|}{ Natural frequencies of bare elastic beam, $\omega_{r}(\mathrm{rad} / \mathrm{sec})$} & \multirow[t]{2}{*}{ Remarks } \\
\hline & & & & \multicolumn{2}{|l|}{$\omega_{1}$} & $\omega_{2}$ & \multicolumn{2}{|l|}{$\omega_{3}$} & $\omega_{4}$ & $\omega_{5}$ & $\omega_{6}$ & \\
\hline \multirow{2}{*}{\multicolumn{2}{|c|}{$\begin{array}{l}\text { MMSM } \\
\text { FEM } \\
\end{array}$}} & & & \multirow{2}{*}{\multicolumn{2}{|c|}{$\begin{array}{l}371.6938 \\
371.6795\end{array}$}} & 1011.2936 & \multicolumn{2}{|c|}{1971.6003} & 3250.7771 & 4849.3266 & 6767.3386 & ${ }^{\mathrm{a}}$ Exact \\
\hline & & & & & & 1011.2537 & \multicolumn{2}{|c|}{1971.5221} & 3250.6491 & 4849.1424 & 6767.1099 & $n_{e}=80$ \\
\hline \multicolumn{13}{|c|}{ (b) Loaded rigid-and-elastic-coupled beam } \\
\hline \multirow[t]{2}{*}{ Cases } & \multicolumn{4}{|c|}{$\begin{array}{l}{ }^{\mathrm{b}} \text { Concentrated } \\
\text { elements }\end{array}$} & \multirow[t]{2}{*}{ Methods } & \multirow{2}{*}{\multicolumn{2}{|c|}{ Rigid-body motions considered? }} & \multicolumn{4}{|c|}{ Natural freqs. of loaded rigid-and-elastic-coupled beam, $\bar{\omega}_{r}(\mathrm{rad} / \mathrm{s})$} & \\
\hline & $m_{i}^{*}$ & $e_{i}^{*}$ & $J_{i}^{*}$ & $k_{t, i}^{*}$ & & & & $\bar{\omega}_{1}\left(\bar{\omega}_{R 1}\right)$ & $\bar{\omega}_{2}\left(\bar{\omega}_{R 2}\right)$ & $\bar{\omega}_{3}\left(\bar{\omega}_{E 1}\right)$ & $\bar{\omega}_{4}\left(\bar{\omega}_{E 2}\right)$ & $\bar{\omega}_{5}\left(\bar{\omega}_{E 3}\right)$ \\
\hline \multirow[t]{4}{*}{1} & \multirow[t]{4}{*}{0.2} & \multirow[t]{4}{*}{0} & \multirow[t]{4}{*}{0} & \multirow[t]{4}{*}{0} & ${ }^{\mathrm{c}} \mathrm{CMSM}$ & \multicolumn{2}{|l|}{ No $\left(n^{\prime}=6\right)$} & - & - & $\begin{array}{l}204.9123 \\
\mathrm{~g}(-3.763 \%)\end{array}$ & $548.4417(-6.076 \%)$ & $1174.4789(-0.851 \%)$ \\
\hline & & & & & ${ }^{\mathrm{d}}$ MMSM & \multicolumn{2}{|l|}{ Yes $\left(n^{\prime}=8\right)$} & $0(0.000 \%)$ & $0(0.000 \%)$ & $213.0365(0.052 \%)$ & $585.0049(0.185 \%)$ & $1188.5136(0.334 \%)$ \\
\hline & & & & & ${ }^{\mathrm{e}}$ Eqs. $(78 \mathrm{a}, \mathrm{b})$ & \multicolumn{2}{|l|}{ Yes } & $0(0.000 \%)$ & $0(0.000 \%)$ & - & - & - \\
\hline & & & & & ${ }^{\mathrm{f}}$ FEM & \multicolumn{2}{|l|}{-} & 0 & 0 & 212.9253 & 583.9231 & 1184.5574 \\
\hline \multirow[t]{4}{*}{2} & \multirow[t]{4}{*}{0.2} & \multirow[t]{4}{*}{0.01} & \multirow[t]{4}{*}{0.2} & \multirow[t]{4}{*}{0} & CMSM & No $\left(n^{\prime}=6\right)$ & & - & - & $204.8738(-4.612 \%)$ & $547.8347(-6.964 \%)$ & $1170.5148(-1.816 \%)$ \\
\hline & & & & & MMSM & Yes $\left(n^{\prime}=8\right)$ & & $0(0.000 \%)$ & $0(0.000 \%)$ & $212.9371(-0.858 \%)$ & $583.8691(-0.844 \%)$ & $1184.1815(-0.669 \%)$ \\
\hline & & & & & Eqs. $(78 \mathrm{a}, \mathrm{b})$ & Yes & & $0(0.000 \%)$ & $0(0.000 \%)$ & - & - & - \\
\hline & & & & & FEM & - & & 0 & 0 & 214.7798 & 588.8421 & 1192.1632 \\
\hline 3 & 0 & 0 & 0 & 1 & CMSM & No $\left(n^{\prime}=6\right)$ & & - & - & $375.7881(0.0013 \%)$ & $1013.0331(0.004 \%)$ & $1972.6116(0.004 \%)$ \\
\hline & & & & & MMSM & Yes $\left(n^{\prime}=8\right)$ & & $36.1323(0.001 \%)$ & $47.8585(0.003 \%)$ & $375.7978(0.004 \%)$ & $1013.0343(0.004 \%)$ & $1972.6117(0.004 \%)$ \\
\hline & & & & & Eqs. $(78 a, b)$ & Yes & & $37.2724(3.156 \%)$ & $47.0896(-1.603 \%)$ & - & - & - \\
\hline & & & & & FEM & - & & 36.1319 & 47.8569 & 375.7831 & 1012.9942 & 1972.5332 \\
\hline 4 & 0.2 & 0.01 & 0.2 & 1 & CMSM & No $\left(n^{\prime}=6\right)$ & & - & - & $207.2458(-4.454 \%)$ & $548.6993(-6.933 \%)$ & $1170.8479(-1.815 \%)$ \\
\hline & & & & & MMSM & Yes $\left(n^{\prime}=8\right)$ & & $25.9482(0.490 \%)$ & $29.4192(-0.588 \%)$ & $215.0288(-0.866 \%)$ & $584.5770(-0.847 \%)$ & $1184.5023\left(-0.670^{\circ}\right.$ \\
\hline & & & & & Eqs. $(78 \mathrm{a}, \mathrm{b})$ & Yes & & $26.3556(2.068 \%)$ & $29.2154(-1.276 \%)$ & - & - & - \\
\hline & & & & & FEM & - & & 25.8217 & 29.5931 & 216.9077 & 589.5717 & 1192.4962 \\
\hline
\end{tabular}

a Obtained from exact solution

b $m_{i}^{*}=m_{i} / m_{\mathrm{ref}}, e_{i}^{*}=e_{i} / L, J_{i}^{*}=J_{i} / J_{\mathrm{ref}}, k_{t, i}^{*}=k_{t, i} / k_{\mathrm{t}, \mathrm{ref}}(i=1 \sim 5)$.

c $\mathrm{CMSM}=$ Conventional MSM

d MMSM = Modified MSM.

e Eqs. (78a,b) based on theory for SDOF spring-mass system shown in Appendix.

${ }^{\mathrm{f}}$ FEM with $n_{e}=80$.

g Percentage differences determined by $\Theta=\left(\bar{\omega}_{r, X}-\bar{\omega}_{r, F E M}\right) \times 100 \% / \bar{\omega}_{r, F E M}($ with $\mathrm{X}=$ MMSM, CMSM or Eqs. $(78 \mathrm{a}, \mathrm{b}))$. 


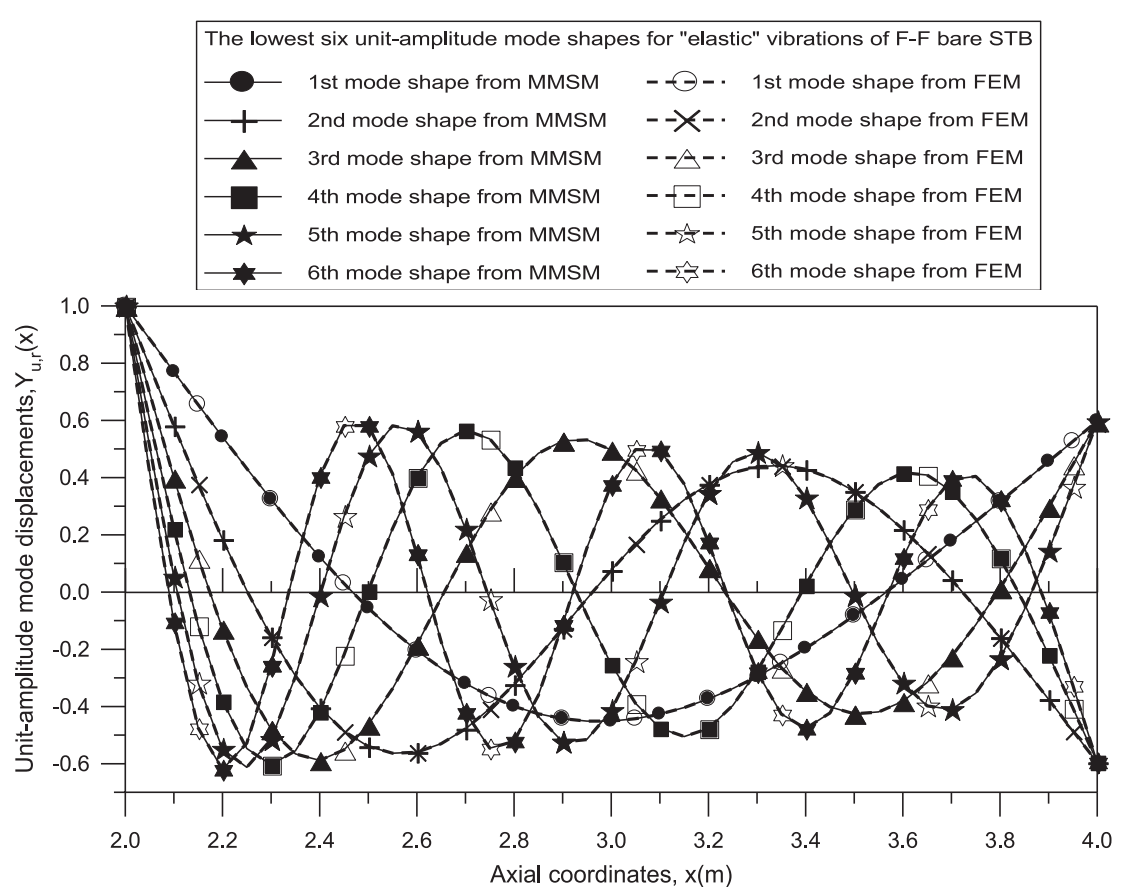

Fig. 6. Lowest six unit-amplitude mode shapes for elastic vibrations of F-F bare STB (Fig. 5) with natural frequencies shown in Table 2(a), as obtained from MMSM (-) and FEM (- - -).

clamped, pinned-pinned or free-clamped) loaded STBs.

\subsection{Free vibration analysis of F-F bare STB}

Since the dimensions of the tapered beam in the previous section resemble those of a trapezoidal plate, the dimensions of the STB studied in this subsection are set as shown in Fig. 5, i.e., $b_{0}=b_{1}=$ $0.03 \mathrm{~m}, h_{0}=0.03 \mathrm{~m}, h_{1}=0.06 \mathrm{~m}, L_{0}=2 \mathrm{~m}, L_{1}=4 \mathrm{~m}$ and $L=L_{1}-L_{0}=2 \mathrm{~m}$. Furthermore, the material constants of the beam are specified as $\rho=$ $7850 \mathrm{~kg} / \mathrm{m}^{3}$ and $E=2.068 \times 10^{11} \mathrm{~N} / \mathrm{m}^{2}$.
In MSM, the normal mode shapes originate from the bare beam. Thus, the lowest six natural frequencies and associated mode shapes for the F-F bare STB are studied first. The results are shown in Table 2(a) for natural frequencies $\omega_{r}(\mathrm{rad} / \mathrm{s}, r=1-$ 6), Fig. 6 for the unit-amplitude (natural) mode shapes and, Fig. 7 for the normal mode shapes. Referring to Table 2(a), it is seen that the values of $\omega_{r}$ $(r=1-6)$ obtained from MMSM are very close to those obtained from FEM. The corresponding unitamplitude mode shapes obtained by the two methods are also in good agreement, as shown in Fig. 6. In other words, the multi-step non-uniform beam shown in Fig. 3 represents an appropriate

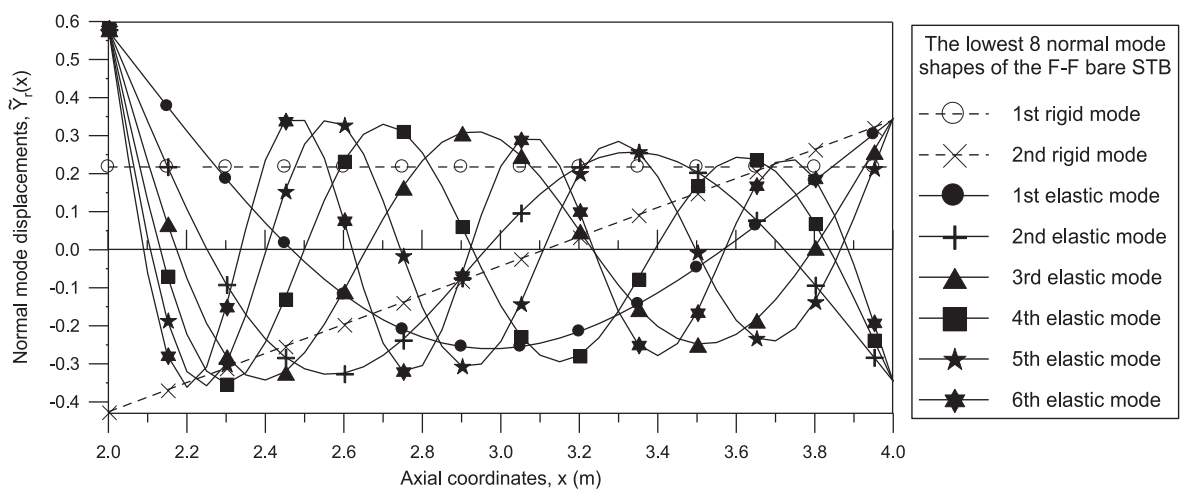

Fig. 7. Lowest eight normal mode shapes for rigid-body motions (- - -) coupled with elastic vibrations (--) of F-F bare STB (Fig. 5) obtained from MMSM. 
discrete model of the continuous STB required for the finite element analysis purposes.

From Eqs. (54) and (55), one sees that the orthonormality conditions for the normal mode shapes of rigid-body motions and elastic vibrations of the F-F bare STB are the key requirements for the MMSM. Thus, the Kronecker delta matrix $[\delta]$ for the lowest eight normal mode shapes of the F-F bare STB is obtained numerically as

In Eq. (76), the 1st and 2nd normal mode shapes are obtained from rigid-body motions of the bare

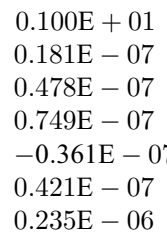

$0.100 \mathrm{E}+01$

$-0.294 \mathrm{E}-07 \quad 0.100 \mathrm{E}+01$

$-0.162 \mathrm{E}-07-0.162 \mathrm{E}-07$

$-0.628 \mathrm{E}-07$

$-0.255 \mathrm{E}-06$

$0.117 \mathrm{E}-05$

$-0.669 \mathrm{E}-07$

$0.469 \mathrm{E}-06$

$-0.235 \mathrm{E}-05$

$0.100 \mathrm{E}+01$

$0.610 \mathrm{E}-07$

$-0.911 \mathrm{E}-06$

$0.361 \mathrm{E}-05$

Referring to Table 2(b), four loading conditions are studied here, namely (1) all of the CEs are equal to zero except $m_{i}^{*}=0.2(i=1 \sim 5) ;(2)$ all of the CEs consisting only of lumped masses with eccentricity and rotary inertia $\left(m_{i}^{*}=0.2, e_{i}^{*}=0.01\right.$ and $\left.J_{i}^{*}=0.2\right)$; (3) all of the CEs are equal to zero except each translational spring with $k_{t, i}^{*}=1.0$; and (4) all of the CEs are attached to the F-F beam with $m_{i}^{*}=0.2$, $e_{i}^{*}=0.01, J_{i}^{*}=0.2$ and $k_{t, i}^{*}=1.0(i=1 \sim 5)$. From Table 2(b) and the Appendix presented at the end of this paper, one sees that:

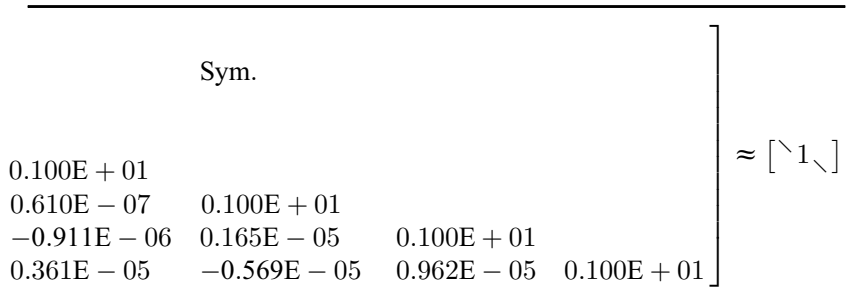

beam, while the 3rd to 8th normal mode shapes are obtained from elastic vibrations, as shown in Fig. 7, in which the two rigid-body mode shapes are denoted by the dashed lines, while the six elastic mode shapes are denoted by the solid lines.

\subsection{Influence of various CEs on F-F loaded STB}

This subsection examines the free vibration characteristics of the previous STB (Fig. 5) carrying 5 identical sets of CEs located at $x_{1}=2.0 \mathrm{~m}$ (node 1), $x_{2}=2.5 \mathrm{~m}$ (node 21$), x_{3}=3.0 \mathrm{~m}$ (node 41$), x_{4}=3.5 \mathrm{~m}$ (node 61) and $x_{5}=4.0 \mathrm{~m}$ (node 81 ). For convenience, the following non-dimensional parameters for the CEs are introduced:

$m_{i}^{*}=m_{i} / m_{\mathrm{ref}}, e_{i}^{*}=e_{i} / L, J_{i}^{*}=J_{i} / J_{\mathrm{ref}}$,
$k_{t, i}=k_{t, i} / k_{\mathrm{t}, \mathrm{ref}}($ for $i=1-5)$

where $m_{\text {ref }}$ and $k_{\mathrm{t}, \text { ref }}$ are the reference mass and reference translational stiffness for each set of CEs and have values of $m_{\mathrm{ref}}=\rho A_{\mathrm{ave}} L=21.1950 \mathrm{~kg}$ and $k_{t, \text { ref }}=E I_{\text {ave }} / L^{3}=5888.953125 \mathrm{~N} / \mathrm{m}$, respectively. Note that $A_{\text {ave }}=b_{0} h_{\text {ave }}$ and $I_{\text {ave }}=b_{0} h_{\text {ave }}^{3} / 12$, where $h_{\text {ave }}=\left(h_{0}+h_{1}\right) / 2$ represents the average height of the STB. In the finite element model, the STB is subdivided into 80 beam elements (i.e., $n=n_{e}=$ 80 ) joined by 81 nodes. Thus, the five sets of CEs are located at nodes 1, 21, 41, 61 and 81 , respectively, as shown in Fig. 5(a).

(a) The lowest two natural frequencies represent those of the rigid-body motions of the F-F loaded STB, while the other three natural frequencies represent those of the elastic vibrations. For convenience, the five frequencies are denoted as $\bar{\omega}_{1}=\bar{\omega}_{R 1}, \bar{\omega}_{2}=\bar{\omega}_{R 2}$, $\bar{\omega}_{3}=\bar{\omega}_{E 1}, \bar{\omega}_{4}=\bar{\omega}_{E 2}$ and $\bar{\omega}_{5}=\bar{\omega}_{E 3}$, where subscripts " $R$ " and " $E$ " represent rigid-body motions and the elastic vibration of the F-F STB, respectively.

(b) From the previous work on the F-F uniform beams presented by [31,32], one finds that the natural frequencies for the rigid-body motion of an elastic F-F loaded beam (i.e., $\bar{\omega}_{R 1}$ and $\bar{\omega}_{R 2}$ ) are very close to those for the heave and pitch motions of an elastically supported rigid F-F loaded beam given by Eqs. (A.1a,b) of the Appendix at the end of this paper, i.e.,

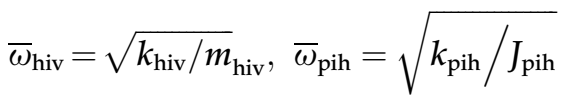

where $k_{\text {hiv }}$ and $m_{\text {hiv }}$ are the effective stiffness and mass, respectively, of the loaded beam for heave motion given by Eqs. (A.2a,b) of the Appendix, while $k_{\text {pih }}$ and $J_{\text {pih }}$ are the effective stiffness and mass moment of inertia, respectively, of the loaded beam for pitch motion given by Eqs. $(\mathrm{A} .3 \mathrm{a}, \mathrm{b})$ of the Appendix. 
The following discussions examine the applicability of Eqs. (78a,b) to the case of a F-F singletapered (non-uniform) beam.

(c) For Case 1 in Table 2(b), the attached CEs consist only of five lumped masses with $m_{i}^{*}=0.2$. Since these masses provide no contribution to the stiffness $k_{\text {hiv }}$ or $k_{\text {pih, }}$ one has $k_{\text {hiv }}=k_{\text {pih }}=0$ and $\bar{\omega}_{\text {hiv }}=\bar{\omega}_{\text {pih }}=0$ according to Eqs. $(78 a, b)$. This finding is consistent with the results obtained from MMSM $\left(\bar{\omega}_{R 1}=\bar{\omega}_{R 2}=0\right)$, as shown in Table 2(b). It is additionally seen in Fig. 2(b) that CMSM provides no information regarding the rigid-body motion of the F-F loaded STB. Furthermore, the maximum percentage difference between the CMSM results and the FEM results $(\epsilon=-6.076 \%)$ is much larger than that between the MMSM results and the FEM results $(\epsilon=0.334 \%)$, where $\epsilon$ is computed as

$\epsilon=\left(\bar{\omega}_{r, X}-\bar{\omega}_{r, F E M}\right) \times 100 \% / \bar{\omega}_{r, F E M}$

in which $\bar{\omega}_{r, F E M}$ is the $r$-th natural frequency of the loaded STB obtained from FEM, and $\bar{\omega}_{r, X}$ (with $X=$ MMSM, CMSM or Eqs. $(78 a, b)$ ) is the corresponding natural frequency obtained from modified MSM (MMSM), conventional MSM (CMSM), or Eqs. (78a,b).

Comparing the results presented in Table 2(b) with those given in Table 2(a), one sees that $\bar{\omega}_{E r}<<\omega_{r}$ (for $r=1,2,3$ ). This is reasonable since the lumped masses, $m_{i}(i=1-5)$, inevitably reduce the natural frequencies of the loaded STB.

(d) For Case 2 in Table 2(b), the attached CEs have the form of only lumped masses, each with $m_{i}^{*}=$ $0.2, e_{i}^{*}=0.01$ and $J_{i}^{*}=0.2$. Since these CEs also provide no contribution to the stiffness $k_{\text {hiv }}$ or $k_{\text {pihh }}$ one again has $k_{\text {hiv }}=k_{\text {pih }}=0$ and $\bar{\omega}_{\text {hiv }}=$ $\bar{\omega}_{\text {pih }}=0$ from Eqs. $(78 \mathrm{a}, \mathrm{b})$. Consequently, $\bar{\omega}_{R 1}=$ $\bar{\omega}_{R 2}=0$, as one may see from Table 2(b). All of the natural frequencies $\bar{\omega}_{r}(r=1-5)$ obtained from MMSM are very close to those obtained from FEM (maximum percentage difference $\epsilon=$ $-0.858 \%$ ). However, for CMSM, the maximum percentage difference is much larger $(\epsilon=-$ $6.964 \%$ ). It is seen that, compared to Case 1 , the values of $\bar{\omega}_{r}(r=1-5)$ for Case 2 are smaller. This result is reasonable since the eccentricities $e_{i}$ and rotary inertias $J_{i}$ of the lumped masses $m_{i}$ $(i=1-5)$ inevitably reduce the natural frequencies of the loaded STB. (e) For Case 3 in Table 2(b), the attached CEs have the form of translational springs, each with $k_{t, i}^{*}=$ 1.0. According to Eqs. (A.2a) and (A.3a) of the Appendix, the contributions of $k_{t, i}(i=1-5)$ to the stiffnesses of the heave and pitch motions are given respectively as

$$
\begin{aligned}
& k_{h i v}=\sum_{i=1}^{5} k_{t, i}(\text { for heave motion }), \\
& k_{\text {pih }}=\sum_{i=1}^{5} k_{t, i}\left(x_{i}-x_{C}\right)^{2}(\text { for pitch motion })
\end{aligned}
$$

$(80 a, b)$

Furthermore, the values of $m_{\text {hiv }}$ and $J_{\text {pih }}$ are given by Eqs. (A.2b) and (A.3b), respectively. Thus, the values of $k_{\text {hiv }}, k_{\text {pih }}, m_{\text {hiv }}$ and $J_{\text {pih }}$ can be obtained from Eqs. (80a,b), (A.2b) and (A.3b) and then substituted into Eqs. $(78 \mathrm{a}, \mathrm{b})$ to give

$$
\begin{aligned}
& \bar{\omega}_{\text {hiv }}=\sqrt{k_{\text {hiv }} / m_{\text {hiv }}}=37.2724 \mathrm{rad} / \mathrm{sec}, \\
& \bar{\omega}_{\text {pih }}=\sqrt{k_{\text {pih }} / J_{\text {pih }}}=47.0896 \mathrm{rad} / \mathrm{sec}
\end{aligned}
$$

Comparing these results with those obtained from MMSM, i.e., $\bar{\omega}_{R 1}=36.1323 \mathrm{rad} / \mathrm{s}$ and $\bar{\omega}_{R 2}=$ $47.8585 \mathrm{rad} / \mathrm{s}$, it is seen that the percentage difference for $\bar{\omega}_{\text {hiv }}$ is $€=3.156 \%$, while that of $\bar{\omega}_{\text {pih }}$ is $€=-1.603 \%$, according to Eq. (79). In other words, Eqs. $(78 \mathrm{a}, \mathrm{b})$ derived for the uniform beam (with $\epsilon<1.0 \%$ ) are also applicable for a non-uniform STB, albeit with a greater percentage difference (e.g., $\epsilon \approx 3.0 \%$ ) due to the effects of the un-symmetrical configuration of the heave and pitch motions of the entire STB with respect to its center of gravity. It is additionally noted that the values of $\bar{\omega}_{r}(r=1-5)$ obtained from MMSM are very close to those obtained from FEM with a maximum percentage difference of just $\epsilon=0.004 \%$.

(f) For Case 4 in Table 2(b), the attached CEs comprise five identical sets of CEs, each consisting of a lumped mass with $m_{i}^{*}=0.2$ (with $e_{i}^{*}=0.01$ and $\left.J_{i}^{*}=0.2\right)$ and a translational spring with $k_{t, i}^{*}=1.0(i=1 \sim 5)$. Referring to Table 2(b), one sees that the natural frequencies for heave and pitch motions obtained from Eqs. $(78 \mathrm{a}, \mathrm{b})$ are given respectively as

$$
\begin{aligned}
& \bar{\omega}_{\text {hiv }}=\sqrt{k_{\text {hiv }} / m_{\text {hiv }}}=26.3556 \mathrm{rad} / \mathrm{sec}, \\
& \bar{\omega}_{\text {pih }}=\sqrt{k_{\text {pih }} / J_{\text {pih }}}=29.2154 \mathrm{rad} / \mathrm{sec}
\end{aligned}
$$

The corresponding values obtained from MMSM are $\bar{\omega}_{R 1}=25.9482 \mathrm{rad} / \mathrm{s}$ and 
(a)

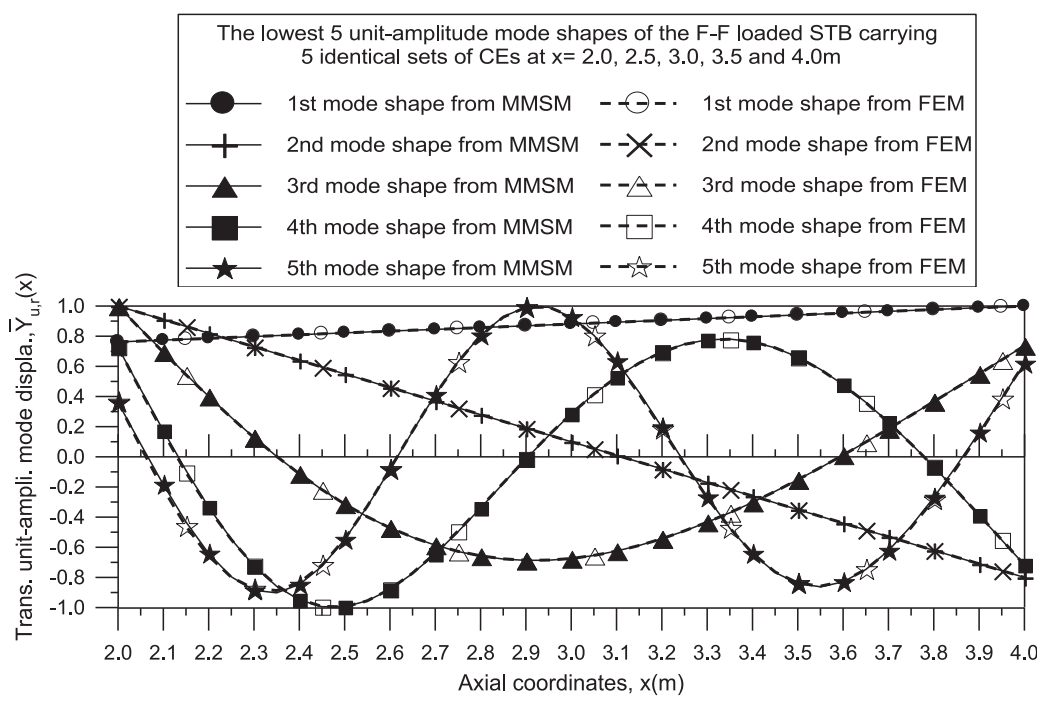

(b)

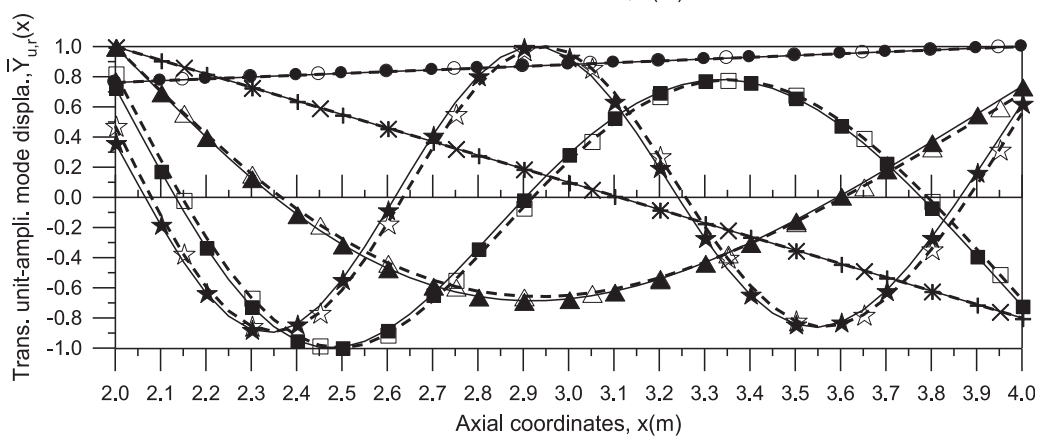

(c)

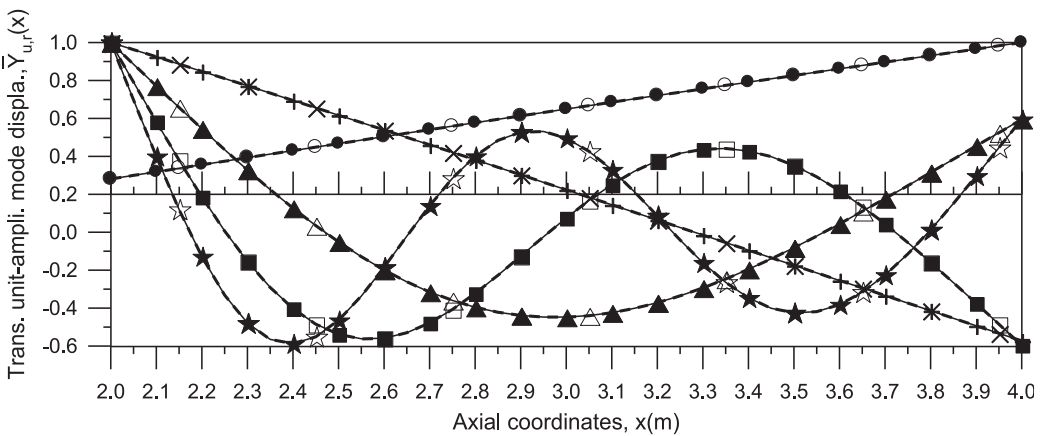

(d)

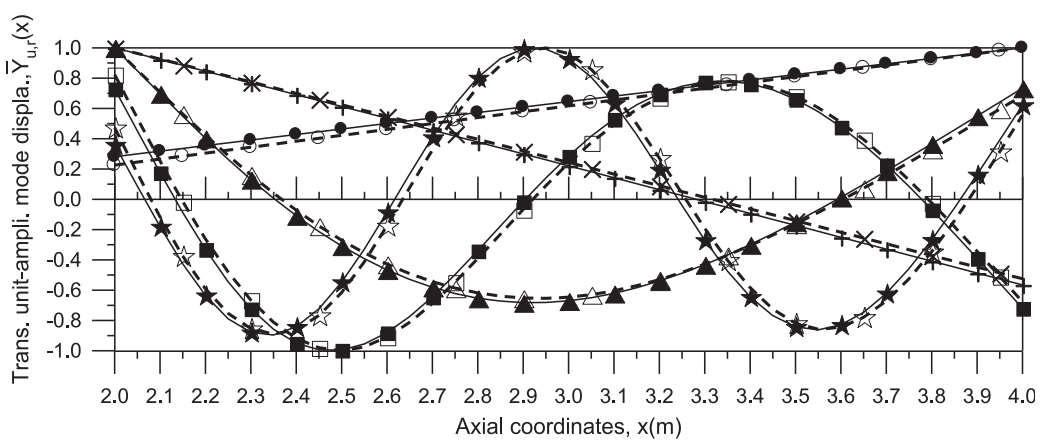

Fig. 8. Lowest five unit-amplitude mode shapes for F-F loaded STB shown in Fig. 5 with: (a) attached lumped masses only (Case 1 in Table 2(b)); (b) attached lumped masses with eccentricities and rotary inertias (Case 2 in Table 2(b)); (c) attached translational springs only (Case 3 in Table 2(b)); (d) attached various CEs (Case 4 in Table 2(b)). Note that the mode shapes obtained from MMSM are denoted by solid lines (--) while those obtained from FEM are denoted by dashed lines $(---)$. 
$\bar{\omega}_{R 2}=29.4192 \mathrm{rad} / \mathrm{s}$, respectively. In other words, the percentage difference of $\bar{\omega}_{\text {hiv }}$ is $€=$ $2.068 \%$, while that of $\bar{\omega}_{\text {pih }}$ is $\epsilon=-1.276 \%$, according to Eq. (79). Furthermore, the values of $\bar{\omega}_{r}(r=1-5)$ obtained from MMSM are very close to those obtained from FEM with a maximum percentage difference of just $\epsilon=-$ $0.866 \%$.

(g) Based on the discussions above and Table 2(b), one finds that the natural frequencies for the heave and pitch motions of the rigid loaded beam $\left(\bar{\omega}_{\text {hiv }}\right.$ and $\left.\bar{\omega}_{\text {pih }}\right)$ obtained from Eqs. $(78 a, b)$ are reasonably close to those for the quasi rigidbody motions of the elastic loaded beam $\left(\bar{\omega}_{R 1}\right.$ and $\bar{\omega}_{R 2}$ ) obtained from the MMSM to some degree. Moreover, the values of $\bar{\omega}_{R 1}$ and $\bar{\omega}_{R 2}$ obtained from MMSM are very close to those obtained from FEM.

(h) A comparison of Tables 2(a) and 2(b) reveals that the lumped masses with (or without) eccentricities and rotary inertias reduce the natural frequencies for elastic vibrations of the loaded beam, while the translational springs increase the natural frequencies. For Case 4 in Table 2(b), the combined effect of all the CEs is to reduce the natural frequencies of the elastic vibration of the loaded beam. However, it is evident that the combined effect of all the CEs is dependent on both the locations of the CEs and their relative magnitudes.

Besides the lowest five natural frequencies of the F-F loaded STB listed in Table 2(b), Figs. 8(a)-(d) show the unit-amplitude mode shapes for Cases $1-4$, respectively. It is seen that the mode shapes obtained from MMSM (shown by the solid lines) are in good agreement with those obtained from FEM (denoted by the dashed lines) in every case.

\section{Conclusions}

1. For a free-free (F-F) bare single-tapered beam (STB), the normal mode shapes for rigid-body motion and elastic vibration satisfy the orthonormality conditions. Consequently, the modified mode-superposition method (MMSM) presented in this paper is capable of determining the lowest several natural frequencies and mode shapes (of rigid-and-elastic-coupled vibrations) of the associated F-F loaded STB carrying multiple various concentrated elements (CEs). By contrast, the conventional mode-superposition method (CMSM) is incapable of solving this problem since it neglects the effects of rigid-body motions of the F-F bare STB in its formulation.

2. The good agreement observed between the results obtained from MMSM and those obtained from FEM indicates that, for the title problem considered in the present study, replacing the rigid-body motion responses of a F-F STB with both the transverse displacement of its center of gravity (c.g.) and the rotational angle about the c.g., and evaluating the elastic vibration response of a continuous STB with that of a discrete multi-step non-uniform beam are the efficient approaches.

3. Previous studies have indicated that, for a uniform beam, the natural frequencies for rigidbody (heave and pitch) motions of a F-F rigid loaded beam $\left(\bar{\omega}_{\text {hiv }}\right.$ and $\left.\bar{\omega}_{\text {pih }}\right)$ obtained from SDOF spring-mass system theory are close to those of a F-F elastic loaded beam $\left(\bar{\omega}_{R 1}\right.$ and $\left.\bar{\omega}_{R 2}\right)$ obtained from MMSM (with a percentage difference $\epsilon<1.0 \%$ ). The same conclusion also applies for the STB problem studied in this paper. However, the percentage difference is larger $(\epsilon \approx 3.0 \%)$ due to the effects of un-symmetrical configuration of the heave and pitch motions of the entire rigid STB with respect to its c.g.

4. For the various CEs studied in this paper, the lumped masses reduce the natural frequencies of the F-F loaded STB, the translational springs increase the natural frequencies, and the combined effects of all the CEs are dependent on the particular locations and magnitudes of the various CEs. Thus, one may change the natural frequencies and mode shapes of the F-F loaded beam to avoid serious vibration responses due to resonance by simply adjusting the distributions and proportions of the different CEs. Therefore, in addition to its theoretical contributions, this study also provides a useful source of reference for practical applications.

5. For the title problem considered in this study, the order of the characteristic equation derived from MMSM, i.e., $n^{\prime}$, is much smaller than that of the equation derived from FEM, i.e., $2(n+1)$. Consequently, the CPU time required by MMSM is far lower than that for FEM, and hence it is much better suited to the development of real-time demonstration technology.

\section{Appendix.}

Natural frequencies for heave and pitch motions of F-F rigid loaded STB 
If the F-F loaded STB is rigid, then the entire loaded beam may be modeled as a single-degree-offreedom (SDOF) spring-mass (S-M) system with its natural frequency for the translational (heave) motion, $\omega_{\text {hiv }}$, and that for the rotational (pitch) motion, $\omega_{\text {pih }}$, determined by $[31,32]$ as

$\bar{\omega}_{\text {hiv }}=\sqrt{k_{\text {hiv }} / m_{\text {hiv }}}, \bar{\omega}_{\text {pih }}=\sqrt{k_{\text {pih }} / J_{\text {pih }}}$

where $k_{\text {hiv }}$ and $m_{\text {hiv }}$ are the effective stiffness and mass for heave motion, respectively, while $k_{\text {pih }}$ and $J_{\text {pin }}$ are the effective stiffness and mass moment of inertia for pitch motion of the loaded STB, respectively. For a F-F loaded STB carrying $n+1$ sets of CEs, where each set of CEs consists of a lumped mass $m_{i}$ (with eccentricity $e_{i}$ and rotary inertia $J_{i}$ ) and a translational spring with stiffness $k_{t, i}$ as shown in Fig. 2, the values of $k_{\text {hiv }}, k_{\text {pih }}, m_{\text {hiv }}$ and $J_{\text {pih }}$ are given by [31,32] as

$$
\begin{aligned}
& k_{\text {hiv }}=\sum_{i=1}^{n+1} k_{t, i}, m_{\text {hiv }}=m_{b}+\sum_{i=1}^{n+1} m_{i} \\
& k_{\text {pih }}=\sum_{i=1}^{n+1} k_{t, i} r_{c i}^{2}, J_{\text {pih }}=J_{b}+\sum_{i=1}^{n+1}\left(m_{i} r_{c i}^{2}+J_{i}+m_{i} e_{i}^{2}\right)
\end{aligned}
$$

In Eqs. (A.3a,b), $r_{c i}$ denotes the distance between the attaching point of the $i$ th set of CEs and the center of gravity (c.g.) of the bare STB, C. Thus, from Fig. 2, one has

$$
r_{c i}=x_{i}-x_{C}
$$

where $x_{C}$ is the axial coordinate of point $C$. Furthermore, in Eqs. (A.2b) and (A.3b), $m_{b}$ and $J_{b}$ denote the mass and mass moment of inertia of the bare STB, respectively, and are given by

$$
\begin{aligned}
m_{b}=\int_{L_{0}}^{L_{1}} \rho A(x) d x & =\int_{\xi_{0}}^{1} \rho A_{1} \xi \cdot L_{1} d \xi=\frac{1}{2} \rho A_{1} L_{1}\left(1-\xi_{0}^{2}\right) \\
& =\frac{1}{2} \rho b L\left(h_{0}+h_{1}\right)
\end{aligned}
$$

$$
\begin{gathered}
J_{b}=\int_{L_{0}}^{L_{1}} \rho A(x) r_{c}^{2} d x=\frac{1}{L_{1}} \rho A_{1} \int_{L_{0}}^{\mathrm{L}_{1}} x\left(x-x_{C}\right)^{2} d x \\
=\rho A_{1} L_{1}^{3}\left[\frac{1}{4}\left(1-\xi_{0}^{4}\right)-\frac{2}{3} \xi_{C}\left(1-\xi_{0}^{3}\right)+\frac{1}{2} \xi_{C}^{2}\left(1-\xi_{0}^{2}\right)\right]
\end{gathered}
$$

where

$r_{c}=x-x_{C}$

From Eqs. (A.2a) and (A.3a), one sees that the translational springs (each with stiffness $k_{t, i}$ ) have contributions to both the heave stiffness $k_{\text {hiv }}$ and the pitch stiffness $k_{\text {pih }}$. Similarly, Eqs. (A.2b) and (A.3b) indicate that the lumped masses $m_{i}$ also have contributions to both the heave mass $m_{\text {hiv }}$ and the pitch mass moment of inertia $J_{\text {pih }}$. However, the eccentricity $e_{i}$ and rotary inertia $J_{i}$ of the lumped masses $m_{i}$ have contributions only to the pitch mass moment of inertia $J_{\text {pih. }}$. It is noted that Eqs. (A.2) and (A.3) are applicable for various loading conditions. For example, if the STB carries $n+1$ lumped masses $m_{i}$ only (each with $e_{i}=0$ and $J_{i}=0$ ), then from Eqs. (A.2) and (A.3), one obtains $k_{\text {hiv }}=0, k_{\text {pih }}=0, m_{\text {hiv }}=$ $m_{b}+\sum_{i=1}^{n+1} m_{i}$ and $J_{\text {pih }}=J_{b}+\sum_{i=1}^{n+1} m_{i} r_{c i}^{2}$. Substitution of the latter parameters into Eqs. (A.1a,b) produces $\bar{\omega}_{\text {hiv }}=\bar{\omega}_{R 1}=0$ and $\bar{\omega}_{\text {pih }}=\bar{\omega}_{R 2}=0$. This result is reasonable since the lumped masses $m_{i}(\mathrm{i}=1-\mathrm{n}+1)$ have no contribution to either the heave stiffness $k_{\text {hiv }}$ or the pitch stiffness $k_{\text {pih }}$.

\section{Conflict of interest}

The author declares that there is no conflict of interest in this study.

\section{References}

[1] Abramovich H, Hamburger O. Vibration of a cantilever Timoshenko beam with a tip mass. J Sound Vib 1991;148(1): $162-70$.

[2] Abrate S. Vibration of non-uniform rods and beams. J Sound Vib 1995;185(4):703-16.

[3] Auciello NM. Transverse vibrations of a linearly tapered cantilever beam with tip mass of rotatory inertia and eccentricity. J Sound Vib 1996;194(1):25-34.

[4] Auciello NM, Maurizi MJ. On the natural vibrations of tapered beams with attached inertia elements. J Sound Vib 1997;199(3):522-30.

[5] Bathe KJ. Finite element procedures in engineering analysis. New York: Prentice-Hall International; 1982.

[6] Clough RW, Penzien J. Dynamics of structures. New York: McGraw-Hill; 1975.

[7] Conway HD, Dubil JF. Vibration frequencies of truncatedcone and wedge beams. ASME J Appl Mech 1965;32(4): 932-4.

[8] Cranch ET, Adler AA. Bending vibrations of variable section beams. ASME J Appl Mech 1956;23(1):103-8.

[9] Craver Jr WL, Jampala P. Transverse vibrations of a linearly tapered cantilever beam with constraining springs. J Sound Vib 1993;166(3):521-9.

[10] Dwight HB. Tables of integrals and other mathematical data. $3^{\text {rd }}$ ed. USA: Dept. of Electrical Machinery, Massachusetts Institute of Technology; 1957.

[11] Faires FD, Burden RL. Numerical methods. Boston: PWS; 1993.

[12] Grossi RO, Aranda A, Bhat RB. Vibration of tapered beams with one end spring hinged and the other end with tip mass. J Sound Vib 1993;160(1):175-8. 
[13] Gupta AK. Vibration of tapered beams. J Struct Eng 1985; 111(1):19-36.

[14] Gürgöze M. A note on the vibration of restrained beams and rods with point masses. J Sound Vib 1984;96(4):461-8.

[15] Heidebrecht AC. Vibration of non-uniform simply-supported beams. ASCE J Eng Mech Div 1967;93(2):1-16.

[16] Hoffmann JA, Wertheimer T. Cantilever beam vibration. J Sound Vib 2000;229(5):1269-76.

[17] Huang TC. The effect of rotatory inertia and of shear deformation on the frequency and normal mode equations of uniform beams with simple end conditions. ASME J Appl Mech 1961;28(4):579-84.

[18] Lau JH. Vibration frequencies and mode shapes for a constrained cantilever. ASME J Appl Mech 1984;51(1):182-7.

[19] Lee TW. Transverse vibrations of a tapered beam carrying a concentrated mass. ASME J Appl Mech 1976;43(2):366-7.

[20] Mabie HH, Rogers CB. Transverse vibration of tapered cantilever beams with end support. J Acoust Soc Am 1968; 44(6):1739-41.

[21] Mabie HH, Rogers CB. Transverse vibrations of doubletapered cantilever beams. J Acoust Soc Am 1972;51(5 part 2): 1771-4.

[22] Mabie HH, Rogers CB. Transverse vibration of doubletapered cantilever beams with end support and with end mass. J Acoust Soc Am 1974;55(5):986-91.

[23] Meirovitch L. Analytical methods in vibrations. London: Macmillan; 1967.

[24] Przemieniecki JS. Theory of matrix structural analysis. New York: McGraw-Hill; 1968.

[25] Rossi RE, Laura PAA, Avalos DR, Larrondo H. Free vibrations of Timoshenko beams carrying elastically mounted concentrated masses. J Sound Vib 1993;165(2):209-23.
[26] Rutenberg A. Vibration frequencies for a uniform cantilever with a rotational constraint at a point. ASME J Appl Mech 1978;45(2):422-3.

[27] Wu CC. Static and dynamic analyses of mountain bikes and their riders. Ph.D. thesis. UK: Department of Mechanical Engineering, University of Glasgow; 2013a.

[28] Wu JS. Analytical and numerical methods for vibration analyses. John Wiley \& Sons, Singapore Pte. Ltd; 2013b.

[29] Wu CC, Ballance D. Static and free vibration analyses of a bike using finite element method. International Journal of Engineering Research \& Science 2015a;1(7):60-86.

[30] Wu CC, Ballance D. A new approach for evaluating the pedalling efficiency of a bike rider in seated cycling by using theory of engineering mechanics. Int J Mater Eng Technol 2015b;14(2):115-50.

[31] Wu CC. Influence of rigid-body motions on free vibration characteristics of a free-free beam carrying arbitrary concentrated elements. Int J Appl Eng Res Dev 2016;6(4):1-22.

[32] $\mathrm{Wu}$ CC. Free vibration analysis of a free-free Timoshenko beam carrying multiple concentrated elements with effect of rigid-body motions considered. J Sound Vib 2019;445: 204-27.

[33] Wu JS, Lin TL. Free vibration analysis of a uniform cantilever beam with point masses by an analytical-and-numericalcombined method. J Sound Vib 1990;136(2):201-13.

[34] Wu JS, Hsieh M. Free vibration analysis of a non-uniform beam with multiple point masses. Struct Eng Mech 2000;9(5): 449-67.

[35] Wu JS, Chen DW. Bending vibration of wedge beams with any number of point masses. J Sound Vib 2003;262(5): 1073-90. 